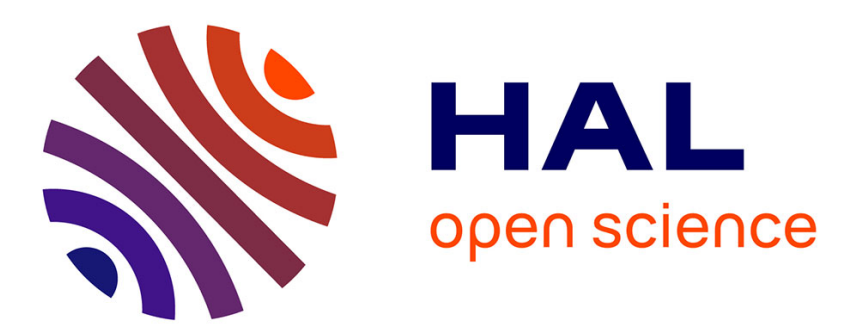

\title{
Modeling of composite plates based on Reissner's Mixed Variational Theorem with variables separation
}

\author{
P. Vidal, L. Gallimard, O. Polit
}

\section{To cite this version:}

P. Vidal, L. Gallimard, O. Polit. Modeling of composite plates based on Reissner's Mixed Variational Theorem with variables separation. Composites Part B: Engineering, 2016, 86, pp.229-242. 10.1016/j.compositesb.2015.09.055 . hal-01366899

\section{HAL Id: hal-01366899 \\ https://hal.science/hal-01366899}

Submitted on 5 Jan 2018

HAL is a multi-disciplinary open access archive for the deposit and dissemination of scientific research documents, whether they are published or not. The documents may come from teaching and research institutions in France or abroad, or from public or private research centers.
L'archive ouverte pluridisciplinaire HAL, est destinée au dépôt et à la diffusion de documents scientifiques de niveau recherche, publiés ou non, émanant des établissements d'enseignement et de recherche français ou étrangers, des laboratoires publics ou privés. 


\title{
Modeling of composite plates based on Reissner's Mixed Variational Theorem with variables separation
}

\author{
P. Vidal*, L. Gallimard, O. Polit \\ LEME - EA 4416, Université Paris Ouest, 50 Rue de Sèvres, 92410, Ville d'Avray, France
}

\section{A B S T R A C T}

In this work, the modeling of laminated composite plates is performed through a variables separation approach based on a Reissner's Variational Mixed Theorem (RMVT). Both the displacement and transverse stress fields are approximated as a sum of separated functions of the in-plane coordinates $x, y$ and the transverse coordinate $z$. This choice yields to a non-linear problem that can be solved by an iterative process. That consists of solving a $2 \mathrm{D}$ and $1 \mathrm{D}$ problem successively at each iteration. In the thickness direction, a fourth-order expansion in each layer is considered. For the in-plane description, classical Finite Element method is used.

Numerical examples involving several representative laminates are addressed to show the accuracy of the present LayerWise (LW) method. It is shown that it can provide quasi-3D results less costly than classical LW computations. In particular, the estimation of the transverse stresses which are of major importance for damage analysis is very good.

\section{Introduction}

Composite and sandwich structures are widely used in the weight-sensitive industrial applications due to their excellent mechanical properties, especially their high specific stiffness and strength. In this context, they can be subjected to severe mechanical loading. For composite design, accurate knowledge of displacements and stresses is required. So, it is important to take into account transverse shear deformation due to the low ratio of transverse shear modulus to axial modulus, or failure due to delamination. In fact, they can play an important role on the behavior of structures in services, which leads to evaluate precisely their influence on local stress fields in each layer, particularly at the interface between layers.

Theoretical models for heterogeneous structures can be classified as follows:

- the Equivalent Single Layer Models (ESLM), where the classical Love-Kirchhoff (CLT [1]), Reissner-Mindlin (FSDT [2]) and higher-order models (HSDT [3-7]) can be found for plates. The

\footnotetext{
* Corresponding author.

E-mail address: philippe.vidal@u-paris10.fr (P. Vidal).
}

first one leads to inaccurate results for composites because both transverse and normal strains are neglected. The second one needs a shear correction factor. Moreover, transverse shear and normal stress continuity conditions at the interfaces between layers are violated for all of them.

- the Layer-Wise Models (LWM) that aim at overcoming the restriction of the ESL. The reader can refer to the works of Pagano [8] and Reddy [9,10]. See also [11-13].

According to Reddy [14], the number of unknowns remains independent of the number of constitutive layers in the ESLM, while the same set of variables is used in each layer for the LWM. Note that excellent reviews and extensive assessments have been made in the following articles [15-20].

Nevertheless, in the framework of the failure analysis of composite structures, the prediction of the interlaminar stresses is of major interest. In particular, the difficulty is to well-describe the interlaminar continuous transverse stresses. Most of the ESLM fail, requiring the use of post-processing treatment [21-23]. Another way is based on the introduction of interface conditions into higher-order model pertaining to the ESLM or to the LWM. This permits to reduce the number of unknowns and can be viewed as Zig-Zag models [24-27]. Unfortunately, for very severe cases, some limitations appear (cf. [28]). 
To overcome these drawbacks, alternative formulations to the displacement-based approach have been developed. For that, the hybrid formulation [29] and partial hybrid formulation [30] have been proposed to improve the accuracy of the transverse shear stresses. It has been extended to a larger domain of applications in Ref. [31] with a three-field Hu-Washizu functional principle. An alternative method is based on the weak compatibility condition on the transverse normal strain. It has been carried out with a zig-zag model in Refs. [32-34] ensuring the continuity of the transverse shear stresses, and with HSDT in Ref. [35] also avoiding the use of the transverse normal stress as unknowns. Nevertheless, the transverse stresses can be introduced as unknowns as in Ref. [36] to derive a mixed Layerwise FE model based on the minimum potential energy principle. Recently, the mixed least-square formulation in conjunction with a FSDT model or LW approach has been successfully applied to laminated plates [37,38]. This formulation seems to have good properties in the framework of Finite Element method, but the number of unknowns can be high as displacements, transverse stresses and in-plane deformations have to be computed. It should be also noted that the mixed approach of Pagano [39] has been employed by Thai et al. [40] for formulating LW finite plate elements.

For the present work, the partially Reissner's Mixed Variational Theorem (RMVT) assuming two independent fields for displacement and transverse stress variables is used in conjunction with a variables separation. The resulting approach ensures a priori interlaminar continuous transverse stress fields. The RMVT approach comes from the works of Reissner, see Refs. [41,42]. It was first applied for multilayered structures in Ref. [43] and then, in Ref. [44] with higher order displacement field and [45] with a Layerwise approach for both displacements and transverse stress fields. Afterward, the approach was widely developed with a systematic approach based on the Carrrera's Unified Formulation to provide a large panels of 2D models for composite structures based on ESL and/or LW descriptions of the unknowns [46-48]. For a further discussion, the reader can refer to [49]. Nevertheless, the LW approach with a RMVT formulation drives to high computational cost. Thus, a promising alternative approach consists of the introduction of the separation of variables which could overcome these drawbacks. Interesting features has been shown in the reduction model framework [50]. So, the aim of the present paper is to assess this particular representation of the unknowns in the framework of a mixed formulation to model laminated and sandwich plates. Thus, both displacements and transverse stresses are written under the form of a sum of products of bidimensional polynomials of $(\mathrm{x}, \mathrm{y})$ and unidimensional polynomials of $\mathrm{z}$. A piecewise fourth-order Lagrange polynomial of $z$ is chosen. As far as the variation with respect to the in-plane coordinates is concerned, a 2D eight-node quadrilateral FE is employed. Using this method, each unknown function of $(\mathrm{x}, \mathrm{y})$ is classically approximated using one degree of freedom (dof) per node of the mesh and the LW unknown functions of $z$ are global for the whole plate. Finally, the deduced non-linear problem implies the resolution of two linear problems alternatively. This process yields to a 2D and a 1D problems in which the number of unknowns is much smaller than a classical Layerwise approach. Note that this type of method has been successfully applied with a displacement-based framework in Refs. [51,52].

We now outline the remainder of this article. First, the RMVT mechanical formulation is described and the separation of the inplane and out-of-plane stresses is introduced. Then, the principles of the PGD are defined in the framework of our study. The particular assumption on the displacements and the transverse stresses yields to a non-linear problem. An iterative process is chosen to solve this one. The FE discretization is also given. Finally, numerical tests are performed for very thick to thin laminated and sandwich plates. Different stacking sequences are also considered. The behavior of the approach are presented and illustrated. The accuracy of the results is assessed with respect to exact reference solutions [53] and results available in open literature. We also focus on the distributions of the transverse stresses along the thickness which are continuous at the interface between adjacent layers. The results issued from the displacement-based approach with a variables separation [52] are given for further assessments.

\section{Reference problem description: the governing equations}

Let us consider a plate occupying the domain $\mathscr{V}=\Omega \times \Omega_{z}$ with $\Omega=[0, a] \times[0, b] \Omega_{z}=[-h / 2, h / 2]$ in a Cartesian coordinate $(x, y, z)$. The plate is defined by an arbitrary region $\Omega$ in the $(x, y)$ plane, located at the midplane for $z=0$, and by a constant thickness $h$. See Fig. 1 .

\subsection{Constitutive relation}

Stresses $\boldsymbol{\sigma}$ and strains $\boldsymbol{\varepsilon}$ are split into two groups:

$\boldsymbol{\sigma}_{p}^{T}=\left[\begin{array}{llll}\sigma_{11} & \sigma_{22} & \sigma_{12}\end{array}\right], \quad \boldsymbol{\sigma}_{n}^{T}=\left[\begin{array}{lll}\sigma_{13} & \sigma_{23} & \sigma_{33}\end{array}\right]$,

$\boldsymbol{\varepsilon}_{p}^{T}=\left[\begin{array}{lll}\varepsilon_{11} & \varepsilon_{22} & \gamma_{12}\end{array}\right], \quad \boldsymbol{\varepsilon}_{n}^{T}=\left[\begin{array}{lll}\gamma_{13} & \gamma_{23} & \varepsilon_{33}\end{array}\right]$

where the subscripts $n$ and $p$ denote transverse and in-plane values, respectively.

The plate can be made of NC perfectly bonded orthotropic layers. Using the separation between transverse and in-plane components, the three dimensional constitutive law of the $k^{\text {th }}$ layer is given by:

$$
\left\{\begin{array}{l}
\boldsymbol{\sigma}_{p H}^{(k)}=\mathbf{Q}_{p p}^{(k)} \boldsymbol{\varepsilon}_{p G}+\mathbf{Q}_{p n}^{(k)} \boldsymbol{\varepsilon}_{n G} \\
\boldsymbol{\sigma}_{n H}^{(k)}=\mathbf{Q}_{n p}^{(k)} \boldsymbol{\varepsilon}_{p G}+\mathbf{Q}_{n n}^{(k)} \boldsymbol{\varepsilon}_{n G}
\end{array}\right.
$$

where

$$
\begin{aligned}
& \mathbf{Q}_{p p}^{(k)}=\left[\begin{array}{lll}
Q_{11}^{(k)} & Q_{12}^{(k)} & Q_{16}^{(k)} \\
Q_{12}^{(k)} & Q_{22}^{(k)} & Q_{26}^{(k)} \\
Q_{16}^{(k)} & Q_{26}^{(k)} & Q_{66}^{(k)}
\end{array}\right] \\
& \mathbf{Q}_{p n}^{(k)}=\mathbf{Q}_{n p}^{(k) T}=\left[\begin{array}{lll}
0 & 0 & Q_{13}^{(k)} \\
0 & 0 & Q_{23}^{(k)} \\
0 & 0 & Q_{36}^{(k)}
\end{array}\right] \quad \mathbf{Q}_{n n}^{(k)}=\left[\begin{array}{ccc}
Q_{55}^{(k)} & Q_{45}^{(k)} & 0 \\
Q_{45}^{(k)} & Q_{44}^{(k)} & 0 \\
0 & 0 & Q_{33}^{(k)}
\end{array}\right]
\end{aligned}
$$

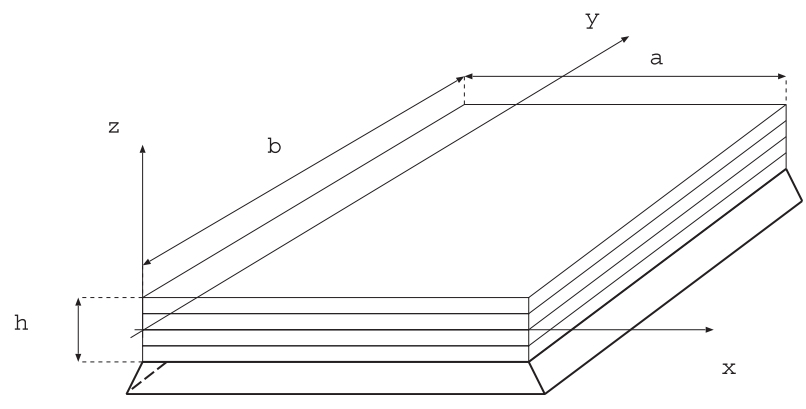

Fig. 1. The laminated plate and coordinate system. 
where $Q_{i j}^{(k)}$ are the three-dimensional stiffness coefficients of the layer $(k)$.

For the involved formulation, the classical Hooke's law is conveniently rewritten under a mixed form:

$\left\{\begin{array}{l}\boldsymbol{\sigma}_{p H}=\mathbf{C}_{p p} \boldsymbol{\varepsilon}_{p G}+\mathbf{C}_{p n} \boldsymbol{\sigma}_{n M} \\ \boldsymbol{\varepsilon}_{n H}=\mathbf{C}_{n p} \boldsymbol{\varepsilon}_{p G}+\mathbf{C}_{n n} \boldsymbol{\sigma}_{n M}\end{array}\right.$

The subscript $G$ indicates that the strain is issued from the geometrical relations, while $H$ means that the quantities are calculated from the Hooke's law. The assumed transverse stresses are denoted as $\boldsymbol{\sigma}_{n M}$. The superscript ${ }^{(k)}$ is omitted for clarity reason.

The relations between the coefficients of the classical Hooke's law Eq. (3) and the mixed one Eq. (4) are:

$$
\begin{array}{ll}
\mathbf{C}_{p p}=\mathbf{Q}_{p p}-\mathbf{Q}_{p n} \mathbf{Q}_{n n}^{-1} \mathbf{Q}_{n p} & \mathbf{C}_{p n}=\mathbf{Q}_{p n} \mathbf{Q}_{n n}^{-1} \\
\mathbf{C}_{n p}=-\mathbf{Q}_{n n}^{-1} \mathbf{Q}_{n p} & \mathbf{C}_{n n}=\mathbf{Q}_{n n}^{-1}
\end{array}
$$

\subsection{The weak form of the boundary value problem}

The formulation of the problem is based on the Reissner's partially Mixed Variational Theorem [41], denoted RMVT. In this formulation, the Principle of Virtual Displacement is modified by introducing the constraint equation to enforce the compatibility of the transverse strain components. This term also depends on the assumed transverse stresses. See also $[17,49]$ in the framework of multi-layered structures. Thus, the problem can be formulated as follows:

Find $\mathbf{u}(M) \in U$ (space of admissible displacements) and $\sigma_{n M}$ such that

$$
\begin{aligned}
\int & \iint_{\mathscr{V}}\left(\delta \boldsymbol{\varepsilon}_{p G}^{T} \boldsymbol{\sigma}_{p H}+\delta \boldsymbol{\varepsilon}_{n G}^{T} \boldsymbol{\sigma}_{n M}+\delta \boldsymbol{\sigma}_{n M}^{T}\left(\boldsymbol{\varepsilon}_{n G}-\boldsymbol{\varepsilon}_{n H}\right)\right) d V \\
= & \iint_{\partial \mathscr{V}_{F}} \delta \mathbf{u} \cdot \mathbf{t} d S
\end{aligned}
$$

where $\mathbf{t}$ is the prescribed surface forces applied on $\partial \mathscr{V}_{F}$. The body force is not considered in this expression.

\section{Application of the separated representation to plates}

In this section, we introduce the application of the variables separation for plate analysis with a mixed formulation. It has been already developed in Ref. [52] in the framework of a displacementbased approach.

\subsection{The displacement, transverse stress and the strain field}

The unknowns of the problem, i.e. the displacement solution $u_{i}(x, y, z)$ and the transverse stresses $\sigma_{i 3}(x, y, z)$ are constructed as the sum of $N$ products of functions of in-plane coordinates and transverse coordinate $(N \in \mathbb{N}$ is the order of the representation)

$$
\begin{aligned}
\mathbf{u} & =\left[\begin{array}{l}
u_{1}(x, y, z) \\
u_{2}(x, y, z) \\
u_{3}(x, y, z)
\end{array}\right]=\sum_{i=1}^{N}\left[\begin{array}{ll}
f_{1}^{i}(z) & v_{1}^{i}(x, y) \\
f_{2}^{i}(z) & v_{2}^{i}(x, y) \\
f_{3}^{i}(z) & v_{3}^{i}(x, y)
\end{array}\right] \\
& =\sum_{i=1}^{N}\left[\begin{array}{l}
f_{1}^{i}(z) \\
f_{2}^{i}(z) \\
f_{3}^{i}(z)
\end{array}\right] \circ\left[\begin{array}{c}
v_{1}^{i}(x, y) \\
v_{2}^{i}(x, y) \\
v_{3}^{i}(x, y)
\end{array}\right]
\end{aligned}
$$

$$
\begin{aligned}
\boldsymbol{\sigma}_{n M} & =\left[\begin{array}{l}
\sigma_{13}(x, y, z) \\
\sigma_{23}(x, y, z) \\
\sigma_{33}(x, y, z)
\end{array}\right]=\sum_{i=1}^{N}\left[\begin{array}{l}
f_{\sigma_{1}}^{i}(z) \tau_{1}^{i}(x, y) \\
f_{\sigma_{2}}^{i}(z) \tau_{2}^{i}(x, y) \\
f_{\sigma_{3}}^{i}(z) \tau_{3}^{i}(x, y)
\end{array}\right] \\
& =\sum_{i=1}^{N}\left[\begin{array}{l}
f_{\sigma_{1}}^{i}(z) \\
f_{\sigma_{2}}^{i}(z) \\
f_{\sigma_{3}}^{i}(z)
\end{array}\right] \circ\left[\begin{array}{c}
\tau_{1}^{i}(x, y) \\
\tau_{2}^{i}(x, y) \\
\tau_{3}^{i}(x, y)
\end{array}\right]
\end{aligned}
$$

where $\left(f_{1}^{i}, f_{2}^{i}, f_{3}^{i}\right),\left(f_{\sigma_{1}}^{i}, f_{\sigma_{2}}^{i}, f_{\sigma_{3}}^{i}\right)$ are defined in $\Omega_{z}$ and $\left(v_{1}^{i}, v_{2}^{i}, v_{3}^{i}\right)$, $\left(\tau_{1}^{i}, \tau_{2}^{i}, \tau_{3}^{i}\right)$ are defined in $\Omega$. The “。” operator is Hadamard's element-wise product.In this paper, a classical eight-node FE approximation is used in $\Omega$ and a LW description is chosen in $\Omega_{z}$ as it is particulary suitable for the modeling of composite structure. The strain derived from Eq. (7) is

$$
\begin{gathered}
\boldsymbol{\varepsilon}_{p G}(u)=\sum_{i=1}^{N}\left[\begin{array}{c}
f_{1}^{i} v_{1,1}^{i} \\
f_{2}^{i} v_{2,2}^{i} \\
f_{1}^{i} v_{1,2}^{i}+f_{2}^{i} v_{2,1}^{i}
\end{array}\right] \\
\boldsymbol{\varepsilon}_{n G}(u)=\sum_{i=1}^{N}\left[\begin{array}{c}
\left(f_{1}^{i}\right)^{\prime} v_{1}^{i}+f_{3}^{i} v_{3,1}^{i} \\
\left(f_{2}^{i}\right)^{\prime} v_{2}^{i}+f_{3}^{i} v_{3,2}^{i} \\
\left(f_{3}^{i}\right)^{\prime} v_{3}^{i}
\end{array}\right]
\end{gathered}
$$

where the prime stands for the classical derivative $\left(f_{i}^{\prime}=\frac{d f_{i}}{d z}\right.$, and ()$, \alpha$ for the partial derivative.

\subsection{The problem to be solved}

The resolution of Eq. (6) is based on a greedy algorithm. If we assume that the first $m$ functions have been already computed, the trial function for the iteration $m+1$ is written as

$\mathbf{u}^{m+1}=\mathbf{u}^{m}+\left[\begin{array}{ll}f_{1} & v_{1} \\ f_{2} & v_{2} \\ f_{3} & v_{3}\end{array}\right]=\mathbf{u}^{m}+\mathbf{f} \circ \mathbf{v}$

$\boldsymbol{\sigma}_{n M}^{m+1}=\boldsymbol{\sigma}_{n M}^{m}+\left[\begin{array}{ll}f_{\sigma_{1}} & \tau_{1} \\ f_{\sigma_{2}} & \tau_{2} \\ f_{\sigma_{3}} & \tau_{3}\end{array}\right]=\boldsymbol{\sigma}_{n M}^{m}+\mathbf{f}_{\sigma} \circ \boldsymbol{\tau}$

where $\left(v_{1}, v_{2}, v_{3}\right),\left(\tau_{1}, \tau_{2}, \tau_{3}\right),\left(f_{1}, f_{2}, f_{3}\right)$ and $\left(f_{\sigma_{1}}, f_{\sigma_{2}}, f_{\sigma_{3}}\right)$ are the functions to be computed and $\mathbf{u}^{m}, \boldsymbol{\sigma}_{n M}^{m}$ are the associated known sets at iteration $m$ defined by

$\mathbf{u}^{m}=\sum_{i=1}^{m}\left[\begin{array}{ll}f_{1}^{i} & v_{1}^{i} \\ f_{2}^{i} & v_{2}^{i} \\ f_{3}^{i} & v_{3}^{i}\end{array}\right] \quad \boldsymbol{\sigma}_{n M}^{m}=\sum_{i=1}^{m}\left[\begin{array}{ll}f_{\sigma_{1}}^{i} & \tau_{1}^{i} \\ f_{\sigma_{2}}^{i} & \tau_{2}^{i} \\ f_{\sigma_{3}}^{i} & \tau_{3}^{i}\end{array}\right]$

The test function is

$$
\begin{aligned}
& \delta\left[\begin{array}{ll}
f_{1} & v_{1} \\
f_{2} & v_{2} \\
f_{3} & v_{3}
\end{array}\right]=\left[\begin{array}{lll}
\delta f_{1} & v_{1}+f_{1} & \delta v_{1} \\
\delta f_{2} & v_{2}+f_{2} & \delta v_{2} \\
\delta f_{3} & v_{3}+f_{3} & \delta v_{3}
\end{array}\right]=\delta \mathbf{f} \circ \mathbf{v}+\delta \mathbf{v} \circ \mathbf{f} \\
& \delta\left[\begin{array}{ll}
f_{\sigma_{1}} \tau_{1} \\
f_{\sigma_{2}} & \tau_{2} \\
f_{\sigma_{3}} & \tau_{3}
\end{array}\right]=\left[\begin{array}{ll}
\delta f_{\sigma_{1}} & \tau_{1}+f_{\sigma_{1}} \delta \tau_{1} \\
\delta f_{\sigma_{2}} & \tau_{2}+f_{\sigma_{2}} \delta \tau_{2} \\
\delta f_{\sigma_{3}} & \tau_{3}+f_{\sigma_{3}} \delta \tau_{3}
\end{array}\right]=\delta \mathbf{f}_{\sigma} \circ \boldsymbol{\tau}+\delta \boldsymbol{\tau} \circ \mathbf{f}_{\sigma}
\end{aligned}
$$


with

$\mathbf{v}=\left[\begin{array}{l}v_{1} \\ v_{2} \\ v_{3}\end{array}\right] \mathbf{f}=\left[\begin{array}{l}f_{1} \\ f_{2} \\ f_{3}\end{array}\right] \boldsymbol{\tau}=\left[\begin{array}{l}\tau_{1} \\ \tau_{2} \\ \tau_{3}\end{array}\right] \mathbf{f}_{\sigma}=\left[\begin{array}{l}f_{\sigma_{1}} \\ f_{\sigma_{2}} \\ f_{\sigma_{3}}\end{array}\right]$

The test functions defined by Eq. (13), Eq. (14), the trial functions defined by Eq. (10), Eq. (11), and the mixed constitutive relation Eq. (4) are introduced into the weak form Eq. (6) to obtain the two following equations:

$$
\begin{gathered}
\int_{\Omega} \int_{\Omega_{z}}\left[\boldsymbol{\varepsilon}_{p G}(f \circ \delta v)^{T}\left(\mathbf{C}_{p p} \boldsymbol{\varepsilon}_{p G}(f \circ v)+\mathbf{C}_{p n} \mathbf{f}_{\sigma} \circ \boldsymbol{\tau}\right)+\boldsymbol{\varepsilon}_{n G}(f \circ \delta v)^{T} \mathbf{f}_{\sigma} \circ \boldsymbol{\tau}\right. \\
\left.+\left(\mathbf{f}_{\sigma} \circ \delta \boldsymbol{\tau}\right)^{T}\left(\boldsymbol{\varepsilon}_{n G}(f \circ v)-\left(\mathbf{C}_{n p} \boldsymbol{\varepsilon}_{p G}(f \circ v)+\mathbf{C}_{n n} \mathbf{f}_{\sigma} \circ \boldsymbol{\tau}\right)\right)\right] d z d \boldsymbol{\Omega} \\
=\int_{\partial \mathscr{V}_{F}}(\mathbf{f} \circ \delta \mathbf{v})^{T} \mathbf{t} d S-\int_{\Omega_{z}}\left[\boldsymbol { \varepsilon } _ { p G } ( f \circ \delta v ) ^ { T } \left(\mathbf{C}_{p p} \boldsymbol{\varepsilon}_{p G}\left(u^{m}\right)\right.\right. \\
\left.+\mathbf{C}_{p n} \boldsymbol{\sigma}_{n M}^{m}\right)+\boldsymbol{\varepsilon}_{n G}(f \circ \delta v)^{T} \boldsymbol{\sigma}_{n M}^{m}+\left(\mathbf{f}_{\sigma} \circ \delta \boldsymbol{\tau}\right)^{T}\left(\boldsymbol{\varepsilon}_{n G}\left(u^{m}\right)\right. \\
\left.\left.\quad-\left(\mathbf{C}_{n p} \boldsymbol{\varepsilon}_{p G}\left(u^{m}\right)+\mathbf{C}_{n n} \boldsymbol{\sigma}_{n M}^{m}\right)\right)\right] d z d \Omega
\end{gathered}
$$

$$
\begin{gathered}
\int_{\Omega_{z}} \int_{\Omega}\left[\boldsymbol{\varepsilon}_{p G}(v \circ \delta f)^{T}\left(\mathbf{C}_{p p} \boldsymbol{\varepsilon}_{p G}(\nu \circ f)+\mathbf{C}_{p n} \boldsymbol{\tau} \circ \mathbf{f}_{\sigma}\right)+\boldsymbol{\varepsilon}_{n G}(v \circ \delta f)^{T} \boldsymbol{\tau} \circ \mathbf{f}_{\sigma}\right. \\
\left.+\left(\boldsymbol{\tau} \circ \delta \mathbf{f}_{\sigma}\right)^{T}\left(\boldsymbol{\varepsilon}_{n G}(v \circ f)-\left(\mathbf{C}_{n p} \boldsymbol{\varepsilon}_{p G}(v \circ f)+\mathbf{C}_{n n} \boldsymbol{\tau} \circ \mathbf{f}_{\sigma}\right)\right)\right] d \Omega d z \\
=\int_{\partial \mathscr{V}_{F}}(\mathbf{v} \circ \delta \mathbf{f})^{T} \mathbf{t} d S-\int_{\Omega_{z}} \int_{\Omega}\left[\boldsymbol { \varepsilon } _ { p G } ( v \circ \delta f ) ^ { T } \left(\mathbf{C}_{p p} \boldsymbol{\varepsilon}_{p G}\left(u^{m}\right)\right.\right. \\
\left.\quad+\mathbf{C}_{p n} \boldsymbol{\sigma}_{n M}^{m}\right)+\boldsymbol{\varepsilon}_{n G}(\nu \circ \delta f)^{T} \boldsymbol{\sigma}_{n M}^{m}+\left(\boldsymbol{\tau} \circ \delta \mathbf{f}_{\sigma}\right)^{T}\left(\boldsymbol{\varepsilon}_{n G}\left(u^{m}\right)\right. \\
\left.\left.\quad-\left(\mathbf{C}_{n p} \boldsymbol{\varepsilon}_{p G}\left(u^{m}\right)+\mathbf{C}_{n n} \boldsymbol{\sigma}_{n M}^{m}\right)\right)\right] d \Omega d z
\end{gathered}
$$

From Eq. (16) and Eq. (17), a coupled non-linear problem is derived. Thus, a non linear resolution strategy has to be used. The simplest one is a fixed point method. An initial function $\mathbf{f}^{(0)}, \mathbf{f}_{\sigma}^{(0)}$ is set, and at each step, the algorithm computes two new pairs $\left(\mathbf{v}^{(k+1)}, \mathbf{f}^{(k+1)}\right),\left(\boldsymbol{\tau}^{(k+1)}, \mathbf{f}_{\sigma}^{(k+1)}\right)$ such that.

- $\mathbf{v}^{(k+1)}, \boldsymbol{\tau}^{(k+1)}$ satisfy Eq. (16) for $\mathbf{f}, \mathbf{f}_{\sigma}$ set to $\mathbf{f}^{(k)}$ and $\mathbf{f}_{\sigma}^{(k)}$

- $\mathbf{f}^{(k+1)}, \mathbf{f}_{\sigma}^{(k+1)}$ satisfy Eq. (17) for $\mathbf{v}, \boldsymbol{\tau}$ set to $\mathbf{v}^{(k+1)}, \boldsymbol{\tau}^{(k+1)}$

These two equations are linear and the first one is solved on $\Omega$, while the second one is solved on $\Omega_{z}$. The fixed point algorithm is stopped when

$\frac{\left\|\mathbf{v}^{(k+1)} \circ \mathbf{f}^{(k+1)}-\mathbf{v}^{(k) \circ \mathbf{f}^{(k)}}\right\|_{\mathscr{V}}}{\left\|\mathbf{v}^{(0)} \circ \mathbf{f}^{(0)}\right\|_{\mathscr{V}}}+\frac{\left\|\boldsymbol{\tau}^{(k+1)} \circ \mathbf{f}_{\sigma}^{(k+1)}-\boldsymbol{\tau}^{(k)} \circ \mathbf{f}_{\sigma}^{(k)}\right\|_{\mathscr{V}}}{\left\|\boldsymbol{\tau}^{(0)} \circ \mathbf{f}_{\sigma}^{(0)}\right\|_{\mathscr{V}}} \leq \varepsilon$

where $\|A\|_{\mathscr{V}}=\left[\int_{\Omega} \int_{\Omega_{z}} \sum_{i=1}^{3} A_{i}^{2} d x d y d z\right]^{1 / 2}$ and $\varepsilon$ is a small parameter to be fixed by the user.

\subsection{Finite element discretization}

To build the plate finite element approximation, a discrete representation of the functions $\left(\mathbf{v}, \tau, \mathbf{f}, \mathbf{f}_{\sigma}\right)$ must be introduced. In this work, a classical finite element approximation in $\Omega$ and $\Omega_{z}$ is used. The elementary vector of degrees of freedom (dof) associated with one element $\Omega_{e}$ of the mesh in $\Omega$ is denoted $\mathbf{q}_{e}^{v \tau}$. And, the elementary vector of dofs associated with one element $\Omega_{z e}$ of the mesh in $\Omega_{z}$ is denoted $\mathbf{q}_{e}^{f f_{\sigma}}$. The displacement fields, the strain fields and the transverse stress fields are determined from the values of $\mathbf{q}_{e}^{v \tau}$ and $\mathbf{q}_{e}^{f f_{\sigma}}$ by

$\begin{array}{lll}\mathbf{v}_{e}=\mathbf{N}_{x y} \mathbf{q}_{e}^{v \tau}, & \mathscr{E}_{v}^{e}=\mathbf{B}_{x y} \mathbf{q}_{e}^{v \tau}, & \boldsymbol{\tau}_{e}=\mathbf{N}_{\sigma x y} \mathbf{q}_{e}^{v \tau} \\ \mathbf{f}_{e}=\mathbf{N}_{z} \mathbf{q}_{e} f_{\sigma}, & \mathscr{E}_{f}^{e}=\mathbf{B}_{z} \mathbf{q}_{e}^{f f_{\sigma}}, & \mathbf{f}_{\sigma e}=\mathbf{N}_{\sigma z} \mathbf{q}_{e} f_{\sigma}\end{array}$

where

$\mathscr{E}_{v}^{e T}=\left[\begin{array}{lllllllll}v_{1} & v_{1,1} & v_{1,2} & v_{2} & v_{2,1} & v_{2,2} & v_{3} & v_{3,1} & v_{3,2}\end{array}\right]$

and

$\mathscr{E}_{f}^{e T}=\left[\begin{array}{llllll}f_{1} & f_{1}^{\prime} & f_{2} & f_{2}^{\prime} & f_{3} & f_{3}^{\prime}\end{array}\right]$

The matrices $\mathbf{N}_{x y}, \mathbf{B}_{x y}, \mathbf{N}_{z}, \mathbf{B}_{z}, \mathbf{N}_{\sigma x y}, \mathbf{N}_{\sigma z}$ contain the interpolation functions, their derivatives and the jacobian components.

\subsection{Finite element problem to be solved on $\Omega$}

For the sake of simplicity, the functions $\mathbf{f}^{(k)}, \mathbf{f}_{\sigma}^{(k)}$ which are assumed to be known, will be denoted $\widetilde{\mathbf{f}}, \widetilde{\mathbf{f}}_{\sigma}$, respectively. And the functions $\mathbf{v}^{(k+1)}, \boldsymbol{\tau}^{(k+1)}$ to be computed will be denoted $\mathbf{v}$ and $\boldsymbol{\tau}$, respectively. The strains and the assumed transverse stress in Eq. (16) are defined as

$$
\begin{gathered}
\boldsymbol{\varepsilon}_{p G}(\widetilde{f} \circ v)=\boldsymbol{\Sigma}_{\mathbf{z}}^{\mathbf{p}}(\widetilde{f}) \mathscr{E}_{\mathbf{v}} \\
\boldsymbol{\varepsilon}_{n G}(\widetilde{f} \circ v)=\boldsymbol{\Sigma}_{\mathbf{z}}^{\mathbf{n}}(\widetilde{f}) \mathscr{E}_{\mathbf{v}} \\
\boldsymbol{\sigma}_{n M}\left(\widetilde{f}_{\sigma} \circ \tau\right)=\boldsymbol{\Sigma}_{\mathbf{z}}^{\boldsymbol{\sigma}_{\mathbf{n}}}\left(\widetilde{f}_{\sigma}\right) \boldsymbol{\tau}
\end{gathered}
$$

with

$\Sigma_{\mathbf{z}} \mathbf{p}(\widetilde{f})=\left[\begin{array}{ccccccccc}0 & \widetilde{f}_{1} & 0 & 0 & 0 & 0 & 0 & 0 & 0 \\ 0 & 0 & 0 & 0 & 0 & \widetilde{f}_{2} & 0 & 0 & 0 \\ 0 & 0 & \widetilde{f}_{1} & 0 & \widetilde{f}_{2} & 0 & 0 & 0 & 0\end{array}\right]$

$\Sigma_{\mathbf{z}} \mathbf{n}(\widetilde{f})=\left[\begin{array}{ccccccccc}\tilde{f}_{1}^{\prime} & 0 & 0 & 0 & 0 & 0 & 0 & \widetilde{f}_{3} & 0 \\ 0 & 0 & 0 & \tilde{f}_{2}^{\prime} & 0 & 0 & 0 & 0 & \widetilde{f}_{3} \\ 0 & 0 & 0 & 0 & 0 & 0 & \tilde{f}_{3}^{\prime} & 0 & 0\end{array}\right]$

$\boldsymbol{\Sigma}_{\mathbf{z}}^{\sigma_{\mathbf{n}}}\left(\widetilde{f}_{\sigma}\right)=\left[\begin{array}{ccc}\widetilde{f}_{\sigma_{1}} & 0 & 0 \\ 0 & \widetilde{f}_{\sigma_{2}} & 0 \\ 0 & 0 & \widetilde{f}_{\sigma_{3}}\end{array}\right]$

The variational problem defined on $\Omega$ from Eq. (16) is

$$
\begin{aligned}
\int_{\Omega} & {\left[\delta \mathscr{E}_{\mathbf{v}}^{T} \mathbf{k}_{z}^{v}(\widetilde{f}) \mathscr{E}_{\mathbf{v}}+\delta \mathscr{E}_{\mathbf{v}}^{T} \mathbf{k}_{z}^{v \sigma}\left(\widetilde{f}, \widetilde{f}_{\sigma}\right) \boldsymbol{\tau}+\delta \boldsymbol{\tau}^{T} \mathbf{k}_{z}^{\sigma v}\left(\widetilde{f}, \widetilde{f}_{\sigma}\right) \mathscr{E}_{\mathbf{v}}\right.} \\
+ & \left.\delta \boldsymbol{\tau}^{T} \mathbf{k}_{z}^{\sigma \sigma}\left(\widetilde{f}_{\sigma}\right) \boldsymbol{\tau}\right] d \Omega \\
= & \int_{\Omega} \delta \mathbf{v}^{T} \mathbf{t}_{z}(\widetilde{f}) d \Omega-\int_{\Omega}\left[\delta \mathscr{E}_{\mathbf{v}}^{T} \boldsymbol{\sigma}_{z}\left(\widetilde{f}, \mathbf{u}^{m}, \boldsymbol{\sigma}_{n M}^{m}\right)\right. \\
& \left.+\delta \boldsymbol{\tau}^{T} \boldsymbol{\varepsilon}_{z}\left(\widetilde{f}_{\sigma}, \mathbf{u}^{m}, \boldsymbol{\sigma}_{n M}^{m}\right)\right] d \Omega
\end{aligned}
$$

with 


$$
\begin{aligned}
& \mathbf{k}_{z}^{v}(\widetilde{f})=\int_{\Omega_{z}} \Sigma_{\mathbf{z}}^{\mathbf{p}}(\widetilde{f})^{T} \mathbf{C}_{p p} \Sigma_{\mathbf{z}}^{\mathbf{p}}(\widetilde{f}) d z \\
& \mathbf{k}_{z}^{v \sigma}\left(\widetilde{f}, \widetilde{f}_{\sigma}\right)=\int_{\Omega_{z}}\left[\boldsymbol{\Sigma}_{\mathbf{z}}^{\mathbf{n}}(\widetilde{f})^{T} \boldsymbol{\Sigma}_{\mathbf{z}}^{\sigma_{\mathbf{n}}}\left(\widetilde{f}_{\sigma}\right)+\boldsymbol{\Sigma}_{\mathbf{z}}^{\mathbf{p}}(\widetilde{f})^{T} \mathbf{C}_{p n} \boldsymbol{\Sigma}_{\mathbf{z}}^{\sigma_{\mathbf{n}}}\left(\widetilde{f}_{\sigma}\right)\right] d z \\
& \mathbf{k}_{z}^{\sigma v}\left(\widetilde{f}, \widetilde{f}_{\sigma}\right)=\int_{\Omega_{z}}\left[\boldsymbol{\Sigma}_{\mathbf{z}}^{\sigma_{\mathbf{n}}}\left(\widetilde{f}_{\sigma}\right)^{T} \boldsymbol{\Sigma}_{\mathbf{z}}^{\mathbf{n}}(\widetilde{f})-\boldsymbol{\Sigma}_{\mathbf{z}}^{\sigma_{\mathbf{n}}}\left(\widetilde{f}_{\sigma}\right)^{T} \mathbf{C}_{n p} \boldsymbol{\Sigma}_{\mathbf{z}}^{\mathbf{p}}(\widetilde{f})\right] d z \\
& \mathbf{k}_{z}^{\sigma \sigma}\left(\widetilde{f}_{\sigma}\right)=-\int_{\Omega_{z}} \boldsymbol{\Sigma}_{\mathbf{z}}^{\sigma_{\mathbf{n}}}\left(\widetilde{f}_{\sigma}\right)^{T} \mathbf{C}_{n n} \boldsymbol{\Sigma}_{\mathbf{z}}^{\sigma_{\mathbf{n}}}\left(\widetilde{f}_{\sigma}\right) d z
\end{aligned}
$$

$$
\begin{aligned}
& \boldsymbol{\varepsilon}_{p G}(\widetilde{v} \circ f)=\boldsymbol{\Sigma}_{\mathbf{x y}}^{\mathbf{p}}(\widetilde{v}) \mathscr{E}_{\mathbf{f}} \\
& \boldsymbol{\varepsilon}_{n G}(\widetilde{v} \circ f)=\boldsymbol{\Sigma}_{\mathbf{x y}}^{\mathbf{n}}(\widetilde{v}) \mathscr{E}_{\mathbf{f}} \\
& \boldsymbol{\sigma}_{n M}\left(\widetilde{\tau} \circ f_{\sigma}\right)=\boldsymbol{\Sigma}_{\mathbf{x y}}^{\boldsymbol{\sigma}_{\mathbf{n}}}(\widetilde{\tau}) \mathbf{f}_{\sigma}
\end{aligned}
$$

with

$$
\begin{aligned}
& \Sigma_{\mathbf{x y}}^{\mathbf{p}}(\widetilde{v})=\left[\begin{array}{cccccc}
\widetilde{v}_{1,1} & 0 & 0 & 0 & 0 & 0 \\
0 & 0 & \widetilde{v}_{2,2} & 0 & 0 & 0 \\
\widetilde{v}_{1,2} & 0 & \widetilde{v}_{2,1} & 0 & 0 & 0
\end{array}\right] \\
& \boldsymbol{\Sigma}_{\mathbf{x y}}^{\mathbf{n}}(\widetilde{v})=\left[\begin{array}{cccccc}
0 & \widetilde{v}_{1} & 0 & 0 & \widetilde{v}_{3,1} & 0 \\
0 & 0 & 0 & \widetilde{v}_{2} & \widetilde{v}_{3,2} & 0 \\
0 & 0 & 0 & 0 & 0 & \widetilde{v}_{3}
\end{array}\right]
\end{aligned}
$$

$\mathbf{t}_{z}(\widetilde{f})=\left.\widetilde{\mathbf{f}} \circ \mathbf{t}\right|_{z=z_{F}}$

$\boldsymbol{\sigma}_{z}\left(\widetilde{f}, \mathbf{u}^{m}, \boldsymbol{\sigma}_{n M}^{m}\right)=\int_{\Omega_{z}}\left[\boldsymbol{\Sigma}_{\mathbf{z}}^{\mathbf{p}}(\widetilde{f})^{T} \mathbf{C}_{p p} \boldsymbol{\varepsilon}_{p G}\left(\mathbf{u}^{m}\right)+\boldsymbol{\Sigma}_{\mathbf{z}}^{\mathbf{n}}(\widetilde{f})^{T} \boldsymbol{\sigma}_{n M}^{m}+\boldsymbol{\Sigma}_{\mathbf{z}}^{\mathbf{p}}(\widetilde{f})^{T} \mathbf{C}_{p n} \boldsymbol{\sigma}_{n M}^{m}\right] d z$

$\boldsymbol{\varepsilon}_{z}\left(\widetilde{f}_{\sigma}, \mathbf{u}^{m}, \boldsymbol{\sigma}_{n M}^{m}\right)=\int_{\Omega_{z}}\left[\boldsymbol{\Sigma}_{\mathbf{z}}^{\sigma_{\mathbf{n}}}\left(\widetilde{f}_{\sigma}\right)^{T}\left(\boldsymbol{\varepsilon}_{n G}\left(\mathbf{u}^{m}\right)-\mathbf{C}_{n p} \boldsymbol{\varepsilon}_{p G}\left(\mathbf{u}^{m}\right)-\mathbf{C}_{n n} \boldsymbol{\sigma}_{n M}^{m}\right)\right] d z$

Note that the units of $\mathbf{C}_{n n}, \mathbf{C}_{n p}, \mathbf{C}_{p n}$ and $\mathbf{C}_{p p}$ are different.

The introduction of the finite element approximation Eq. (19) in the variational Eq. (24) leads to the linear system

$\mathbf{K}_{z}\left(\widetilde{f}, \widetilde{f}_{\sigma}\right) \mathbf{q}^{v \sigma}=\mathscr{R}_{v}\left(\widetilde{f}, \widetilde{f}_{\sigma}, \mathbf{u}^{m}, \boldsymbol{\sigma}_{n M}^{m}\right)$

where

- $\mathbf{q}^{v \sigma}$ is the vector of the nodal displacements/transverse stresses associated with the finite element mesh in $\Omega$,

- $\mathbf{K}_{z}\left(\widetilde{f}, \widetilde{f}_{\sigma}\right)$ is the stiffness matrix obtained by summing the elements' stiffness matrices $\mathbf{K}_{z}^{e}\left(\widetilde{f}, \widetilde{f}_{\sigma}\right)=\int\left[\mathbf{B}_{x y}^{T} \mathbf{k}_{z}^{v}(\widetilde{f}) \mathbf{B}_{x y}\right.$ $\left.+\mathbf{B}_{x y}^{T} \mathbf{k}_{z}^{v \sigma}\left(\widetilde{f}, \widetilde{f}_{\sigma}\right) \mathbf{N}_{\sigma x y}+\mathbf{N}_{\sigma x y}^{T} \mathbf{k}_{z}^{\sigma v}\left(\widetilde{f}, \widetilde{f}_{\sigma}\right) \mathbf{B}_{x y}+\mathbf{N}_{\sigma x y}^{T} \mathbf{k}_{z}^{\sigma \sigma}\left(\widetilde{f}_{\sigma}\right) \mathbf{N}_{\sigma x y}\right] d \Omega_{e}$

- $\mathscr{R}_{v}\left(\widetilde{f}, \widetilde{f}_{\sigma}, \mathbf{u}^{m}, \boldsymbol{\sigma}_{n M}^{m}\right)$ is the equilibrium residual obtained by summing the elements' residual load vectors $\mathscr{R}_{v}^{e}\left(\widetilde{f}, \widetilde{f}_{\sigma}, \mathbf{u}^{m}, \boldsymbol{\sigma}_{n M}^{m}\right)=$ $\int_{\Omega_{e}}\left[\mathbf{N}_{x y}^{T} \mathbf{t}_{z}(\widetilde{f})-\mathbf{B}_{x y}^{T} \boldsymbol{\sigma}_{z}\left(\widetilde{f}, \mathbf{u}^{m}, \boldsymbol{\sigma}_{n M}^{m}\right)-\mathbf{N}_{\sigma x y}^{T} \boldsymbol{\varepsilon}_{z}\left(\widetilde{f}_{\sigma}, \mathbf{u}^{m}, \boldsymbol{\sigma}_{n M}^{m}\right)\right] d \Omega_{e}$

\subsection{Finite element problem to be solved on $\Omega_{z}$}

For the sake of simplicity, the functions $\mathbf{v}^{(k+1)}, \boldsymbol{\tau}^{(k+1)}$ which are assumed to be known, will be denoted $\widetilde{\mathbf{v}}, \widetilde{\boldsymbol{\tau}}$ and the functions $\mathbf{f}^{(k+1)}$, $\mathbf{f}_{\sigma}^{(k+1)}$ to be computed will be denoted $\mathbf{f}, \mathbf{f}_{\sigma}$. The strain in Eq. (17) is defined as
$\Sigma_{\mathbf{x y}}^{\sigma_{\mathbf{n}}}(\widetilde{\tau})=\left[\begin{array}{lll}\widetilde{\tau}_{1} & 0 & 0 \\ 0 & \widetilde{\tau}_{2} & 0 \\ 0 & 0 & \tilde{\tau}_{3}\end{array}\right]$

The variational problem defined on $\Omega_{z}$ from Eq. (17) is

$$
\begin{aligned}
& \int_{\Omega_{z}}\left[\delta \mathscr{E}_{\mathbf{f}}^{T} \mathbf{k}_{x y}^{f}(\widetilde{v}) \mathscr{E}_{\mathbf{f}}+\delta \mathscr{E}_{\mathbf{f}}^{T} \mathbf{k}_{x y}^{f f_{\sigma}}(\widetilde{v}, \widetilde{\tau}) \mathbf{f}_{\sigma}+\delta \mathbf{f}_{\sigma}^{T} \mathbf{k}_{x y}^{f_{\sigma} f}(\widetilde{v}, \widetilde{\tau}) \mathscr{E}_{\mathbf{f}}\right. \\
& \left.+\delta \mathbf{f}_{\sigma}^{T} \mathbf{k}_{x y}^{f_{f} f_{\sigma}}(\widetilde{\tau}) \mathbf{f}_{\sigma}\right] d z=\left.\delta \mathbf{f}^{T} \mathbf{t}_{x y}(\widetilde{v})\right|_{z=z_{F}} \\
& -\int_{\Omega_{z}}\left[\delta \mathscr{E}_{\mathbf{f}}^{T} \boldsymbol{\sigma}_{x y}\left(\widetilde{v}, \mathbf{u}^{m}, \boldsymbol{\sigma}_{n M}^{m}\right)+\delta \mathbf{f}_{\sigma}^{T} \boldsymbol{\varepsilon}_{x y}\left(\widetilde{\tau}, \mathbf{u}^{m}, \boldsymbol{\sigma}_{n M}^{m}\right)\right] d z
\end{aligned}
$$

with

$$
\begin{aligned}
& \mathbf{k}_{x y}^{f}(\widetilde{v})=\int_{\Omega} \boldsymbol{\Sigma}_{\mathbf{x y}}^{\mathbf{p}}(\widetilde{v})^{T} \mathbf{C}_{p p} \Sigma_{\mathbf{x y}}^{\mathbf{p}}(\widetilde{v}) d \Omega \\
& \mathbf{k}_{x y}^{f f_{\sigma}}(\widetilde{v}, \widetilde{\tau})=\int_{\Omega}\left[\Sigma_{\mathbf{x y}}^{\mathbf{n}}(\widetilde{v})^{T} \boldsymbol{\Sigma}_{\mathbf{x y}}^{\sigma_{\mathbf{n}}}(\widetilde{\tau})+\Sigma_{\mathbf{x y}}^{\mathbf{p}}(\widetilde{v})^{T} \mathbf{C}_{p n} \boldsymbol{\Sigma}_{\mathbf{x y}}^{\boldsymbol{\sigma}_{\mathbf{n}}}(\widetilde{\tau})\right] d \Omega \\
& \mathbf{k}_{x y}^{f_{f} f}(\widetilde{v}, \widetilde{\tau})=\int_{\Omega}\left[\boldsymbol{\Sigma}_{\mathbf{x y}}^{\sigma_{\mathbf{n}}}(\widetilde{\tau})^{T} \boldsymbol{\Sigma}_{\mathbf{x y}}^{\mathbf{n}}(\widetilde{v})-\Sigma_{\mathbf{x y}}^{\sigma_{\mathbf{n}}}(\widetilde{\tau})^{T} \mathbf{C}_{n p} \boldsymbol{\Sigma}_{\mathbf{x y}}^{\mathbf{p}}(\widetilde{v})\right] d \Omega \\
& \mathbf{k}_{x y}^{f_{f} f_{\sigma}}(\widetilde{\tau})=-\int_{\Omega} \Sigma_{\mathbf{x y}}^{\sigma_{\mathbf{n}}}(\widetilde{\tau})^{T} \mathbf{C}_{n n} \Sigma_{\mathbf{x y}}^{\sigma_{\mathbf{n}}}(\widetilde{\tau}) d \Omega
\end{aligned}
$$

and

$$
\begin{aligned}
& \mathbf{t}_{x y}(\widetilde{v})=\int_{\Omega} \widetilde{\mathbf{v}} \circ \mathbf{t} d \Omega \\
& \boldsymbol{\sigma}_{x y}\left(\widetilde{v}, \mathbf{u}^{m}, \boldsymbol{\sigma}_{n M}^{m}\right)=\int_{\Omega}\left[\Sigma_{\mathbf{x y}}^{\mathbf{p}}(\widetilde{v})^{T} \mathbf{C}_{p p} \boldsymbol{\varepsilon}_{p G}\left(\mathbf{u}^{m}\right)+\boldsymbol{\Sigma}_{\mathbf{x y}}^{\mathbf{n}}(\widetilde{v})^{T} \boldsymbol{\sigma}_{n M}^{m}+\boldsymbol{\Sigma}_{\mathbf{x y}}^{\mathbf{p}}(\widetilde{v})^{T} \mathbf{C}_{p n} \boldsymbol{\sigma}_{n M}^{m}\right] d \Omega \\
& \boldsymbol{\varepsilon}_{x y}\left(\widetilde{\tau}, \mathbf{u}^{m}, \boldsymbol{\sigma}_{n M}^{m}\right)=\int_{\Omega}\left[\boldsymbol{\Sigma}_{\mathbf{x y}}^{\boldsymbol{\sigma}_{\mathbf{n}}}(\widetilde{\tau})^{T}\left(\boldsymbol{\varepsilon}_{n G}\left(\mathbf{u}^{m}\right)-\mathbf{C}_{n p} \boldsymbol{\varepsilon}_{p G}\left(\mathbf{u}^{m}\right)-\mathbf{C}_{n n} \boldsymbol{\sigma}_{n M}^{m}\right)\right] d \boldsymbol{\Omega}
\end{aligned}
$$


The introduction of the finite element discretization Eq. (19) in the variational Eq. (32) leads to the linear system

$\mathbf{K}_{x y}(\widetilde{v}, \widetilde{\tau}) \mathbf{q}^{f f_{\sigma}}=\mathscr{R}_{f}\left(\widetilde{v}, \widetilde{\tau}, \mathbf{u}^{m}, \boldsymbol{\sigma}_{n M}^{m}\right)$

where

- $\mathbf{q}^{f f_{\sigma}}$ is the vector of degree of freedom associated with the F.E. approximations in $\Omega_{z}$,

- $\mathbf{K}_{x y}(\widetilde{v}, \widetilde{\tau})$ is obtained by summing the elements' stiffness matrices:

$$
\begin{aligned}
\mathbf{K}_{x y}^{e}(\widetilde{v}, \widetilde{\tau})= & \int_{\Omega_{z e}}\left[\mathbf{B}_{z}^{T} \mathbf{k}_{x y}^{f}(\widetilde{v}) \mathbf{B}_{z}+\mathbf{B}_{z}^{T} \mathbf{k}_{x y}^{f f_{\sigma}}(\widetilde{v}, \widetilde{\tau}) \mathbf{N}_{\sigma z}+\mathbf{N}_{\sigma z}^{T} \mathbf{k}_{x y}^{f_{\sigma} f}(\widetilde{v}, \widetilde{\tau}) \mathbf{B}_{z}\right. \\
& \left.+\mathbf{N}_{\sigma z}^{T} \mathbf{k}_{x y}^{f_{\sigma} f_{\sigma}}(\widetilde{\tau}) \mathbf{N}_{\sigma z}\right] d z_{e}
\end{aligned}
$$

- $\mathscr{R}_{f}\left(\widetilde{v}, \widetilde{\tau}, \mathbf{u}^{m}, \boldsymbol{\sigma}_{n M}^{m}\right)=\mathscr{R}_{f}^{F}(\widetilde{v})-\mathscr{R}_{f}^{\operatorname{Coup}}\left(\widetilde{v}, \widetilde{\tau}, \mathbf{u}^{m}, \boldsymbol{\sigma}_{n M}^{m}\right)$ is a equilibrium residual with $\mathscr{R}_{f}^{F}(\widetilde{v})=\left.\mathbf{N}_{z}^{T} \mathbf{t}_{x y}(\widetilde{v})\right|_{z=z_{F}}$ and $\mathscr{R}_{f}^{\text {Coup }}\left(\widetilde{v}, \widetilde{\tau}, \mathbf{u}^{m}, \boldsymbol{\sigma}_{n M}^{m}\right)$ is obtained by the summation of the elements' residual vectors given by

$$
\int_{\Omega_{z e}}\left[\mathbf{B}_{z}^{T} \boldsymbol{\sigma}_{x y}\left(\widetilde{v}, \mathbf{u}^{m}, \boldsymbol{\sigma}_{n M}^{m}\right)+\mathbf{N}_{\sigma z}^{T} \boldsymbol{\varepsilon}_{x y}\left(\widetilde{\tau}, \mathbf{u}^{m}, \boldsymbol{\sigma}_{n M}^{m}\right)\right] d z_{e}
$$

\section{Numerical results}

In this section, the present mixed approach is assessed through different static tests. First, a convergence study is carried out to determine the suitable mesh for the further analysis. Then, some comparisons with results available in open literature are provided. They are described below:

LM4, LD4 It refers to the systematic work of Carrera and his "Carrera's Unified Formulation" (CUF), see Refs. [49,18,54]. In particular, a LayerWise model based on a RMVT approach (LM4) or a displacement-based approach (LD4) where each component is expanded until the fourth-order is given. $24 N C+6$ and $12 N C+3$ unknown functions per node are used in this kinematic for LM4 and LD4, respectively.

Jing \& Liao 1989 A partial hybrid stress element based on the Hellinger-Reissner principle is developed [30]. The three displacements and the transverse shear stresses are the independent unknowns. The number of unknowns depends on the number of layers.

Liou \& Sun 1987 A hybrid multilayered element is developed [55]. The 3 components of the displacements and all stresses are interpolated. The number of unknowns depends on the number of layers.

Reddy 1984 A higher-order shear deformation theory is employed with a parabolic distribution of the transverse shear strains through the thickness [4]. The number of unknowns is the same as the FSDT model.

Wu et al., 2005 A global-local higher-order shear deformation theory is carried out in Ref. [56]. It drives to a zig-zag model where the number of unknowns is independent of the number of layers. The continuity of the transverse shear stresses are satisfied.

Then, different types of composites are considered with different geometries. Finally, the behavior of the method is shown on a test involving localized phenomena. The assessments are performed by using exact solutions computed following [53].

In the numerical examples, an eight-node quadrilateral $\mathrm{FE}$ based on the classical Serendipity interpolation functions is used for the unknowns depending on the in-plane coordinates. A Gaussian numerical integration with $3 \times 3$ points is used to evaluate the elementary matrices. As far as the integration with respect to the transverse coordinate is concerned, an analytical integration is performed.

\subsection{Convergence study}

First, the convergence properties are studied so as to derive the suitable mesh refinement for the subsequent numerical examples. A simply supported thin symmetric composite plates is considered from Pagano [53]. The following data are considered:

geometry: composite cross-ply plate $\left(0^{\circ} / 90^{\circ} / 0^{\circ}\right), a=b$ and length-to-thickness ratio $S=20\left(S=\frac{a}{h}\right)$; a quarter of the plate is meshed. All layers have the same thickness.

boundary conditions: simply supported plate subjected to bisinusoidal pressure on the top surface $q(x, y, z=h / 2)=$ $q_{0} \sin \frac{\pi x}{a} \sin \frac{\pi y}{b}$.

material properties:

$$
\begin{array}{ll}
E_{L}=172.4 \mathrm{GPa} & E_{T}=6.895 \mathrm{GPa}, G_{L T}=3.448 \mathrm{GPa} \\
G_{T T}=1.379 \mathrm{GPa}, & \nu_{L T}=\nu_{T T}=0.25
\end{array}
$$

where $L$ refers to the fiber direction, $T$ refers to the transverse direction.

results displacements and stresses are made non-dimensional according to

$$
\begin{aligned}
& \bar{u}(z)=U_{1}(0, b / 2, z) \frac{E_{T}}{q_{0} h S^{3}}, \quad \bar{v}(z)=U_{2}(a / 2,0, z) \frac{E_{T}}{q_{0} h S^{3}}, \\
& \bar{w}(z)=U_{3}(a / 2, b / 2, z) \frac{100 E_{L}}{q_{0} h S^{4}}, \quad \bar{\sigma}_{\alpha \alpha}(z)=\sigma_{\alpha \alpha}(a / 2, b / 2, z) \frac{1}{q_{0} S^{2}}, \\
& \bar{\sigma}_{12}(z)=\sigma_{12}(0,0, z) \frac{1}{q_{0} S^{2}}, \quad \bar{\sigma}_{13}(z)=\sigma_{13}(0, b / 2, z) \frac{1}{q_{0} S} \\
& \bar{\sigma}_{23}(z)=\sigma_{23}(a / 2,0, z) \frac{1}{q_{0} S}, \quad \bar{\sigma}_{33}(z)=\sigma_{33}(a / 2, b / 2, z) \frac{1}{q_{0}}
\end{aligned}
$$

reference values the three-dimensional exact elasticity results are obtained as in Ref. [53].

Different mesh refinements from $N_{x}=N_{y}=2$ to $N_{x}=N_{y}=32$ are considered, $N_{x}$ and $N_{y}$ being the number of elements along the $x$ and $y$ direction, respectively. The results are summarized in Table 1 for both displacements and stresses. Only one couple is built. It appears that the convergence rate is high. For the displacements and the in-plane stresses, a $N_{x}=N_{y}=4$ is sufficient to obtain very accurate results (error rate less than $0.11 \%$ for $\bar{u}, \bar{v}, \bar{w}$, and $1.3 \%$ for $\bar{\sigma}_{\alpha \beta}$ ). It should be noted that the convergence rate of the transverse normal stress is also very high. The transverse shear stress $\bar{\sigma}_{23}$ seems to be the most difficult to estimate with high accuracy. It is about four times smaller than $\bar{\sigma}_{13}$ and the contribution of this component in the total strain energy is the lowest.

Based on all these results, a $N_{x}=N_{y}=24$ mesh is necessary to obtain an error rate of less than $1 \%$. It will be used in the following for the modeling of composite plates.

\subsection{Comparison with results available in literature}

The same example as in the previous section (Section 4.1) is considered with different slenderness ratios. The results are 
Table 1

Convergence study - three layers $\left(0^{\circ} / 90^{\circ} / 0^{\circ}\right)-b=a-N_{z}=N C-\mathrm{S}=20$.

\begin{tabular}{|c|c|c|c|c|c|c|c|c|c|c|}
\hline$N_{x}=N_{y}$ & & $\bar{u}(h / 2)$ & $\bar{v}(-h / 2)$ & $\bar{w}(0)$ & $\bar{\sigma}_{11}(-h / 2)$ & $\bar{\sigma}_{22}(h / 2)$ & $\bar{\sigma}_{12}(h / 2)$ & $\bar{\sigma}_{13}(0)$ & $\bar{\sigma}_{23}(0)$ & $\bar{\sigma}_{33}(h / 2)$ \\
\hline \multirow[t]{2}{*}{2} & present & -0.0069 & 0.0081 & 0.5121 & 0.5716 & -0.2022 & -0.0247 & 0.4435 & 0.1689 & 0.9236 \\
\hline & error & $0.50 \%$ & $1.20 \%$ & $0.83 \%$ & $3.47 \%$ & $3.76 \%$ & $5.83 \%$ & $15.31 \%$ & $80.07 \%$ & $7.64 \%$ \\
\hline \multirow[t]{2}{*}{4} & present & -0.0069 & 0.0080 & 0.5161 & 0.5583 & -0.2092 & -0.0237 & 0.4014 & 0.1222 & 0.9957 \\
\hline & error & $0.05 \%$ & $0.11 \%$ & $0.06 \%$ & $1.06 \%$ & $0.46 \%$ & $1.31 \%$ & $4.38 \%$ & $30.36 \%$ & $0.43 \%$ \\
\hline \multirow[t]{2}{*}{8} & present & -0.0069 & 0.0080 & 0.5164 & 0.5540 & -0.2103 & -0.0234 & 0.3890 & 0.1018 & 1.0035 \\
\hline & error & $0.00 \%$ & $0.01 \%$ & $0.00 \%$ & $0.29 \%$ & $0.07 \%$ & $0.32 \%$ & $1.13 \%$ & $8.53 \%$ & $0.35 \%$ \\
\hline \multirow[t]{2}{*}{16} & present & -0.0069 & 0.0080 & 0.5164 & 0.5528 & -0.2102 & -0.0234 & 0.3857 & 0.0958 & 1.0016 \\
\hline & error & $0.00 \%$ & $0.00 \%$ & $0.00 \%$ & $0.08 \%$ & $0.05 \%$ & $0.08 \%$ & $0.29 \%$ & $2.21 \%$ & $0.16 \%$ \\
\hline \multirow[t]{2}{*}{24} & present & -0.0069 & 0.0080 & 0.5164 & 0.5526 & -0.2102 & -0.0234 & 0.3851 & 0.0947 & 1.0008 \\
\hline & error & $0.00 \%$ & $0.00 \%$ & $0.00 \%$ & $0.03 \%$ & $0.03 \%$ & $0.04 \%$ & $0.13 \%$ & $1.00 \%$ & $0.08 \%$ \\
\hline \multirow[t]{3}{*}{32} & present & -0.0069 & 0.0080 & 0.5164 & 0.5525 & -0.2102 & -0.0234 & 0.3849 & 0.0943 & 1.0005 \\
\hline & error & $0.00 \%$ & $0.00 \%$ & $0.00 \%$ & $0.02 \%$ & $0.02 \%$ & $0.02 \%$ & $0.07 \%$ & $0.56 \%$ & $0.05 \%$ \\
\hline & exact & -0.0069 & 0.0080 & 0.5164 & 0.5524 & -0.2101 & -0.0234 & 0.3846 & 0.0938 & 1. \\
\hline
\end{tabular}

Table 2

Comparison with results available in literature - three layers $\left(0^{\circ} / 90^{\circ} / 0^{\circ}\right)-b=a$.

\begin{tabular}{|c|c|c|c|c|c|c|c|c|}
\hline S & Model & $\bar{w}(0)$ & $\bar{\sigma}_{11}(h / 2)$ & $\bar{\sigma}_{22}(h / 6)$ & $\bar{\sigma}_{12}(h / 2)$ & $\bar{\sigma}_{13}(0)$ & $\bar{\sigma}_{23}(0)$ & $\bar{\sigma}_{33}(h / 2)$ \\
\hline \multirow[t]{4}{*}{4} & present & 2.0059 & 0.8011 & 0.5340 & -0.0511 & 0.2563 & 0.2175 & 1.0003 \\
\hline & [Reddy 1984] & 1.922 & 0.7345 & - & - & - & 0.1832 & - \\
\hline & [Wu et al., 2005] & 2.0557 & 0.8435 & 0.5610 & -0.0522 & 0.2569 & 0.2205 & - \\
\hline & exact & 2.0059 & 0.8008 & 0.5340 & -0.0511 & 0.2559 & 0.2172 & 1.0000 \\
\hline \multirow[t]{8}{*}{10} & present & 0.7530 & 0.5908 & 0.2845 & -0.0288 & 0.3575 & 0.1231 & 1.0003 \\
\hline & [LM4] & 0.7528 & 0.5801 & 0.2796 & -0.0296 & 0.3626 & 0.1249 & - \\
\hline & [LD4] & 0.7528 & 0.5801 & 0.2796 & -0.0296 & 0.3724 & 0.1623 & - \\
\hline & [Liou \& Sun 1987] & 0.7546 & 0.580 & 0.285 & -0.0283 & 0.367 & 0.127 & - \\
\hline & [Jing \& Liao 1989] & 0.7531 & 0.5884 & 0.2834 & -0.0288 & 0.3627 & 0.1284 & - \\
\hline & [Reddy 1984] & 0.713 & 0.5684 & - & - & - & 0.1033 & - \\
\hline & [Wu et al., 2005] & 0.7624 & 0.6331 & 0.2851 & -0.0291 & 0.3540 & 0.1257 & - \\
\hline & exact & 0.7530 & 0.5906 & 0.2845 & -0.0288 & 0.3573 & 0.1228 & 1.0000 \\
\hline \multirow[t]{3}{*}{20} & present & 0.5164 & 0.5526 & 0.2092 & -0.0234 & 0.3851 & 0.0947 & 1.0008 \\
\hline & [Wu et al., 2005] & 0.5194 & 0.5826 & 0.2041 & -0.0234 & 0.3771 & 0.0944 & - \\
\hline & exact & 0.5164 & 0.5524 & 0.2092 & -0.0234 & 0.3846 & 0.0938 & 1.0000 \\
\hline
\end{tabular}

summarized in Table 2. Other approaches available in open literature are given for further comparison. Some of them are based on a displacement formulation (Reddy 1984, Wu et al., 2005, LD4), whereas different components of the stresses are considered as unknowns in the other ones (Jing \& Liao 1989, Liou \& Sun 1987, LM4). As described below, the number of unknowns is either constant (Reddy 1984, Wu et al., 2005) or increasing with the number of layers (Jing \& Liao 1989, Liou \& Sun 1987, LM4, LD4). We can notice that the results of the present approach are in excellent agreement with the reference solution regardless of the slenderness ratios. As expected, the LayerWise approaches give better results than the ESL approaches, especially for the stresses. Indeed, the maximum error rate is $15.6 \%$ for Reddy 1984 and $5.5 \%$ for Wu et al., 2005. Moreover, we observe that the results of the present approach are close to the LM4 results.

\subsection{Anti-symmetric rectangular plate}

In this section, an anti-symmetric thin to very thick plate is considered. The configuration is described as follows:

geometry: composite cross-ply plate $\left(0^{\circ} / 90^{\circ} / 0^{\circ} / 90^{\circ}\right), b=3 a$ and $S=2$ to 40 ; a quarter of the plate is meshed. All layers have the same thickness.

boundary conditions: simply supported plate subjected to bi-sinusoidal pressure on the top surface $q(x, y, z=h / 2)=$ $q_{0} \sin \frac{\pi x}{a} \sin \frac{\pi y}{b}$.

material properties: same material properties as in Section 4.1.

mesh: $N_{x}=N_{y}=24$ is used for the quarter of the plate.

number of dofs: $N d o f_{x y}=10950$ and $N d o f_{z}=24 \times N C+6=102$

results displacements and stresses are made non-dimensional as in Section 4.1 reference values the three-dimensional exact elasticity results are obtained as in Ref. [53].

For this test case, only one couple is needed to recover the solution, as it is not considered as a severe case. Numerical results are given in Table 3. It can be inferred from this table that the accuracy of the results are very good regardless of the slenderness of the structure. In particular, the transverse shear stress $\bar{\sigma}_{13}$ and the transverse normal stress drives to an error rate less than $0.7 \%$.

For further comparisons, the distributions of the in-plane and transverse displacements and stresses through the thickness are shown in Figs. 2-4 for a very thick plate. It is a discriminating test for the classical or refined ESL approach. The results of the present method are in excellent agreement with the exact solution. We can notice that a zig-zag effect occurs for the in-plane displacements. We can also see that the transverse displacement is not constant through the thickness. The a priori continuity conditions of the transverse stresses are exactly satisfied by assumption. The boundary conditions on the upper and lower surfaces are also wellsatisfied whereas they are not constrained. The vertical tangent on the top and bottom of the plate for $\bar{\sigma}_{33}$ is also well-represented.

\subsection{Square sandwich plate}

Another type of composite is now considered to assess the mixed approach. The test is described as follows:

geometry: square sandwich plate with length-to-thickness ratios $S \in\{2,4,10,40\}$. The thickness of each face sheet is $\frac{h}{10}$.

boundary conditions: simply-supported plate subjected to bisinusoidal pressure $q(x, y)=q_{0} \sin \frac{\pi x}{a} \sin \frac{\pi y}{b}$.

material properties: The material of the face sheet is the same as in Section 4.1. The core material is transversely isotropic with 
Table 3

Four layers $\left(0^{\circ} / 90^{\circ} / 0^{\circ} / 90^{\circ}\right)-b=3 a-N_{x}=N_{y}=24-N_{z}=N C$.

\begin{tabular}{|c|c|c|c|c|c|c|c|c|c|c|}
\hline $\mathrm{S}$ & Model & $\bar{u}(h / 2)$ & $\bar{v}(-h / 2)$ & $\bar{w}(0)$ & $\bar{\sigma}_{11}(-h / 2)$ & $\bar{\sigma}_{22}(h / 2)$ & $\bar{\sigma}_{12}(h / 2)$ & $\bar{\sigma}_{13}(0)$ & $\bar{\sigma}_{23}(0)$ & $\bar{\sigma}_{33}(h / 2)$ \\
\hline \multirow[t]{3}{*}{2} & present & -0.0848 & 0.0356 & 10.5470 & -2.0505 & 0.6421 & -0.0742 & 0.3313 & 0.1056 & 1.0000 \\
\hline & error & $0.00 \%$ & $0.00 \%$ & $0.00 \%$ & $0.04 \%$ & $0.03 \%$ & $0.04 \%$ & $0.68 \%$ & $0.10 \%$ & $0.00 \%$ \\
\hline & exact & -0.0848 & 0.0356 & 10.5472 & -2.0497 & 0.6419 & -0.0742 & 0.3290 & 0.1055 & 1.0000 \\
\hline \multirow[t]{3}{*}{4} & present & -0.0438 & 0.0199 & 3.9272 & -1.4541 & 0.3401 & -0.0401 & 0.4554 & 0.0790 & 1.0001 \\
\hline & error & $0.00 \%$ & $0.00 \%$ & $0.00 \%$ & $0.03 \%$ & $0.03 \%$ & $0.04 \%$ & $0.11 \%$ & $0.08 \%$ & $0.01 \%$ \\
\hline & exact & -0.0438 & 0.0199 & 3.9271 & -1.4536 & 0.3400 & -0.0400 & 0.4549 & 0.0790 & 1.0000 \\
\hline \multirow[t]{3}{*}{10} & present & -0.0254 & 0.0099 & 1.5891 & -1.1159 & 0.1741 & -0.0223 & 0.5337 & 0.0482 & 1.0004 \\
\hline & error & $0.00 \%$ & $0.00 \%$ & $0.00 \%$ & $0.03 \%$ & $0.04 \%$ & $0.04 \%$ & $0.08 \%$ & $0.30 \%$ & $0.04 \%$ \\
\hline & exact & -0.0254 & 0.0099 & 1.5891 & -1.1156 & 0.1740 & -0.0223 & 0.5333 & 0.0480 & 1.0000 \\
\hline \multirow[t]{3}{*}{40} & present & -0.0213 & 0.0073 & 1.1173 & -1.0389 & 0.1299 & -0.0179 & 0.5568 & 0.0401 & 1.0048 \\
\hline & error & $0.00 \%$ & $0.00 \%$ & $0.00 \%$ & $0.03 \%$ & $0.01 \%$ & $0.04 \%$ & $0.77 \%$ & $4.04 \%$ & $0.48 \%$ \\
\hline & exact & -0.0213 & 0.0073 & 1.1173 & -1.0386 & 0.1299 & -0.0179 & 0.5525 & 0.0385 & 1.0000 \\
\hline
\end{tabular}
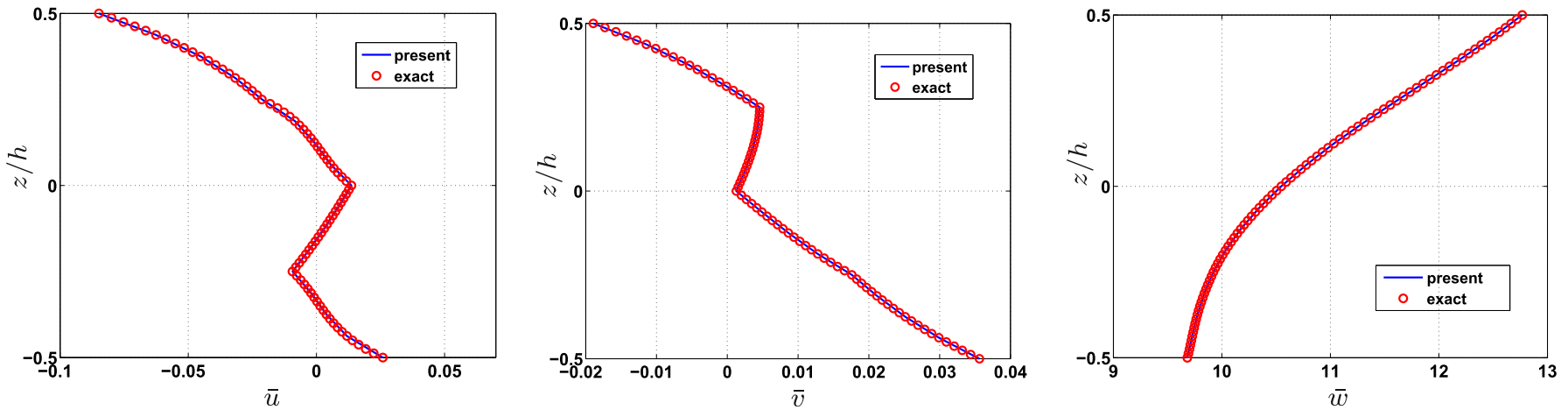

Fig. 2. Distribution of $\bar{u}$ (left), $\bar{v}$ (middle) and $\bar{w}$ (right) along the thickness $-\mathrm{S}=2-4$ layers.
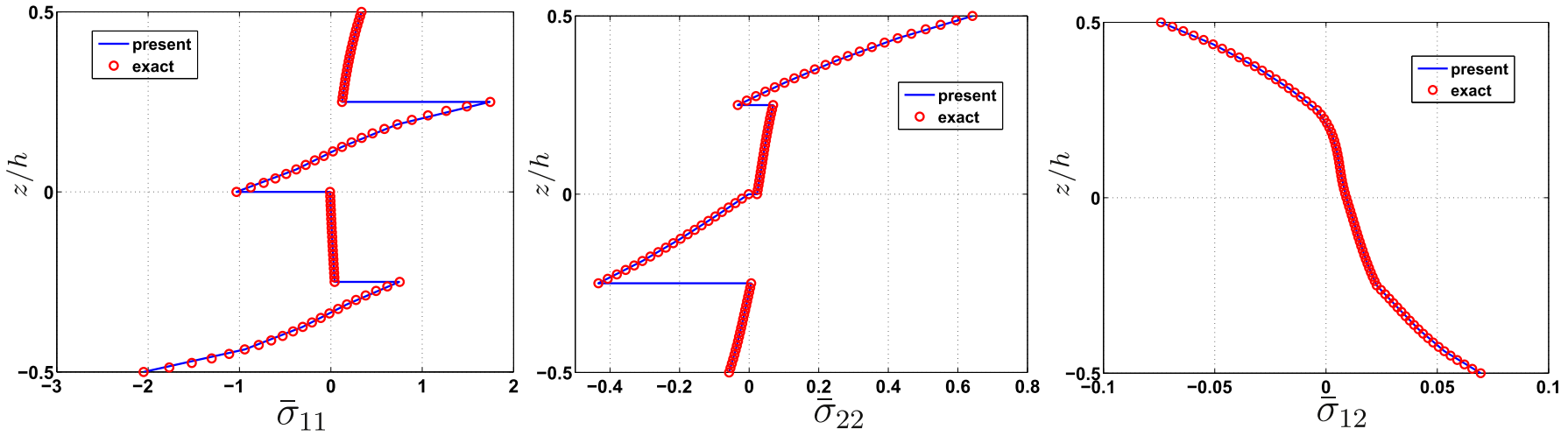

Fig. 3. Distribution of $\bar{\sigma}_{11}$ (left), $\bar{\sigma}_{22}$ (middle) and $\bar{\sigma}_{12}$ (right) along the thickness $-\mathrm{S}=2-4$ layers.
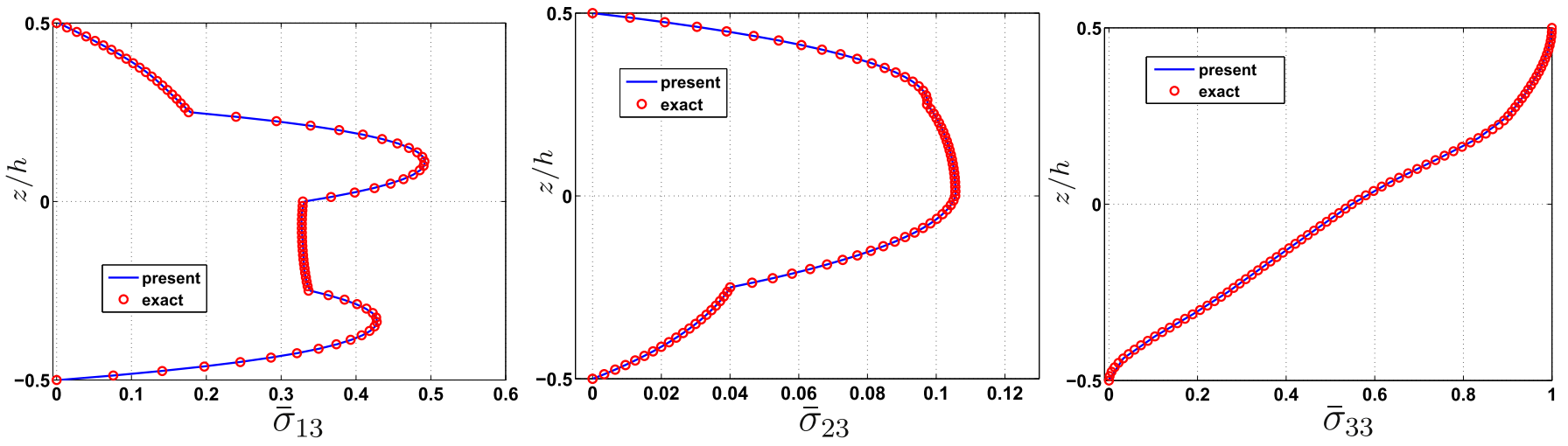

Fig. 4. Distribution of $\bar{\sigma}_{13}$ (left) and $\bar{\sigma}_{23}$ (right) along the thickness - S $=2-4$ layers. 
respect to $z$ and is characterized by: $E_{x x}=E_{y y}=0.04 \mathrm{GPa}$, $E_{z z}=0.5 \mathrm{GPa}, G_{x z}=G_{y z}=0.06 \mathrm{GPa}, G_{x y}=0.016 \mathrm{GPa}, \nu_{x z}=\nu_{y z}=0.02$, $\nu_{x y}=0.25$

mesh: $N_{x}=N_{y}=24$ is used for the quarter of the plate.

number of dofs: $N d o f_{x y}=10950$ and $N d o f_{z}=24 \times N C+6=78$

results displacements and stresses are made non-dimensional as in Section 4.1.

reference values the three-dimensional exact elasticity results are obtained as in Ref. [53].

Again, only one couple is sufficient for this configuration. The results are shown in Table 4 . The approach is also suitable to model very thick to thin sandwich structures with an error rate of less than $0.23 \%$, except for $\bar{\sigma}_{23}$ with an error rate of $2.37 \%$. Figs. 5-7 represent the displacements and the stresses along the thickness for the very thick plate $S=2$. The accuracy of the results are excellent when compared with the exact solutions. Due to the very thick structure and the consideration of the load on the upper surface, some features which are well-described by the present approach can be observed:

- The zig-zag effect of $\bar{u}$ is well-captured with a non-linear variation through the core.

Table 4

Sandwich plate $-b=a-N_{x}=N_{y}=24-N_{z}=N C$.

\begin{tabular}{|c|c|c|c|c|c|c|c|c|c|c|c|}
\hline $\mathrm{S}$ & Model & $\bar{u}(h / 2)$ & $\bar{v}(-h / 2)$ & $\bar{w}(0)$ & $\bar{\sigma}_{11}(-h / 2)$ & $\bar{\sigma}_{22}(h / 2)$ & $\bar{\sigma}_{12}(h / 2)$ & $\bar{\sigma}_{13}(0)$ & $\bar{\sigma}_{13 \max }$ & $\bar{\sigma}_{23}(0)$ & $\bar{\sigma}_{33}(h / 2)$ \\
\hline \multirow[t]{4}{*}{2} & present & -0.0395 & 0.1163 & 22.103 & 3.2793 & 0.4519 & -0.2404 & 0.1849 & 0.3202 & 0.1399 & 1.0003 \\
\hline & error & $0.00 \%$ & $0.00 \%$ & $0.00 \%$ & $0.04 \%$ & $0.03 \%$ & $0.04 \%$ & $0.05 \%$ & $0.05 \%$ & $0.06 \%$ & $0.03 \%$ \\
\hline & exact & -0.0395 & 0.1163 & 22.103 & 3.2781 & 0.4517 & -0.2403 & 0.1848 & 0.3201 & 0.1399 & 1.0000 \\
\hline & LM4 & - & - & 22.103 & 3.2430 & 0.4537 & - & 0.1897 & - & 0.144 & - \\
\hline \multirow[t]{4}{*}{4} & present & -0.0188 & 0.0758 & 7.5963 & 1.5564 & 0.2596 & -0.1437 & 0.2387 & 0.2388 & 0.1073 & 1.0001 \\
\hline & error & $0.00 \%$ & $0.00 \%$ & $0.00 \%$ & $0.03 \%$ & $0.03 \%$ & $0.04 \%$ & $0.03 \%$ & $0.03 \%$ & $0.06 \%$ & $0.01 \%$ \\
\hline & exact & -0.0188 & 0.0758 & 7.5962 & 1.5558 & 0.2595 & -0.1437 & 0.2387 & 0.2387 & 0.1072 & 1.0000 \\
\hline & LM4 & - & - & 7.5947 & 1.5425 & 0.2582 & - & 0.2459 & - & 0.1143 & - \\
\hline \multirow[t]{4}{*}{10} & present & -0.0143 & 0.0313 & 2.2004 & 1.1535 & 0.1105 & -0.0707 & 0.2999 & 0.2999 & 0.0528 & 1.0002 \\
\hline & error & $0.00 \%$ & $0.00 \%$ & $0.00 \%$ & $0.03 \%$ & $0.03 \%$ & $0.04 \%$ & $0.04 \%$ & $0.04 \%$ & $0.21 \%$ & $0.02 \%$ \\
\hline & exact & -0.0143 & 0.0313 & 2.2004 & 1.1531 & 0.1104 & -0.0707 & 0.2998 & 0.2998 & 0.0527 & 1.0000 \\
\hline & LM4 & - & - & 2.2001 & 1.1323 & 0.1093 & - & 0.3042 & - & 0.05354 & - \\
\hline \multirow[t]{3}{*}{40} & present & -0.0138 & 0.0151 & 0.9665 & 1.1005 & 0.0584 & -0.0453 & 0.3232 & 0.3232 & 0.0319 & 1.0008 \\
\hline & error & $0.00 \%$ & $0.00 \%$ & $0.00 \%$ & $0.04 \%$ & $0.01 \%$ & $0.04 \%$ & $0.23 \%$ & $0.23 \%$ & $2.37 \%$ & $0.08 \%$ \\
\hline & exact & -0.0138 & 0.0151 & 0.9665 & 1.1001 & 0.0584 & -0.0453 & 0.3225 & 0.3225 & 0.0312 & 1.0000 \\
\hline
\end{tabular}
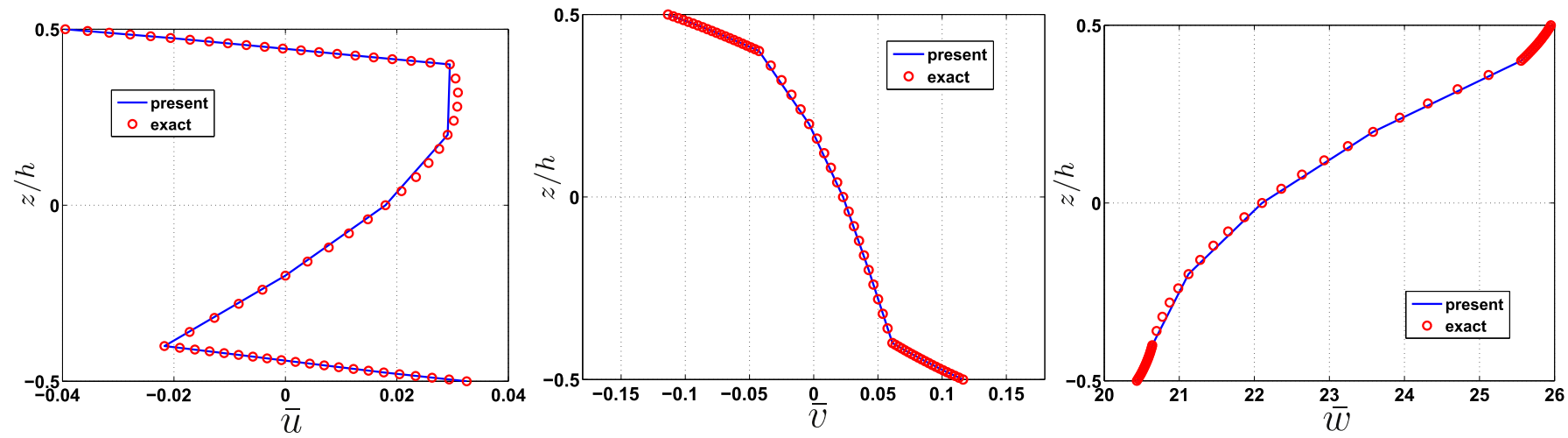

Fig. 5. Distribution of $\bar{u}$ (left), $\bar{v}$ (middle) and $\bar{w}$ (right) along the thickness $-\mathrm{S}=2$ - sandwich.
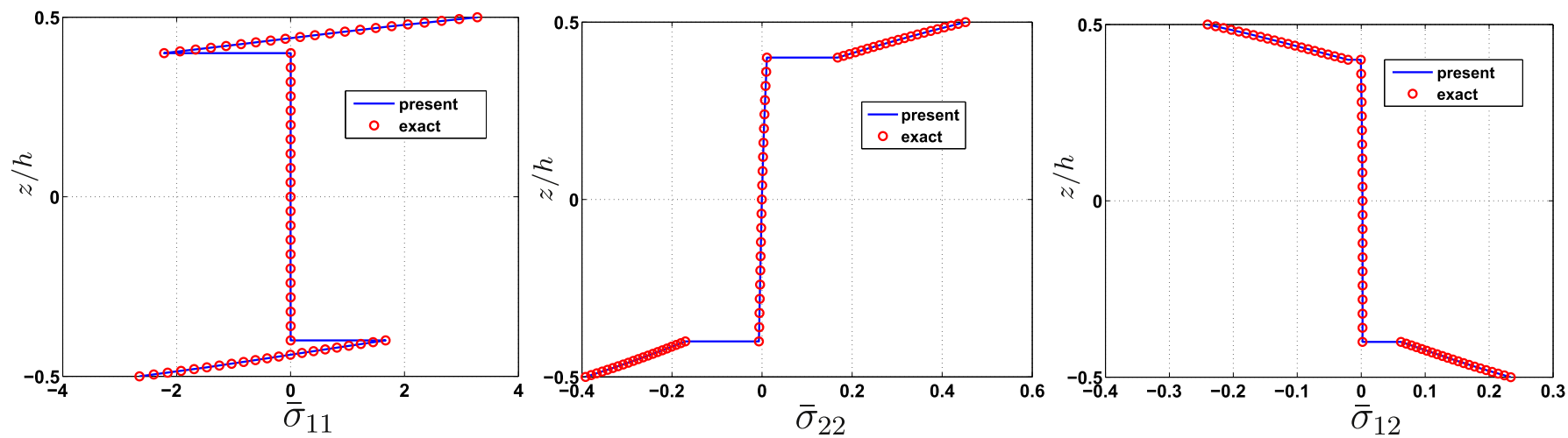

Fig. 6. Distribution of $\bar{\sigma}_{11}$ (left), $\bar{\sigma}_{22}$ (middle) and $\bar{\sigma}_{12}$ (right) along the thickness $-\mathrm{S}=2$ - sandwich. 

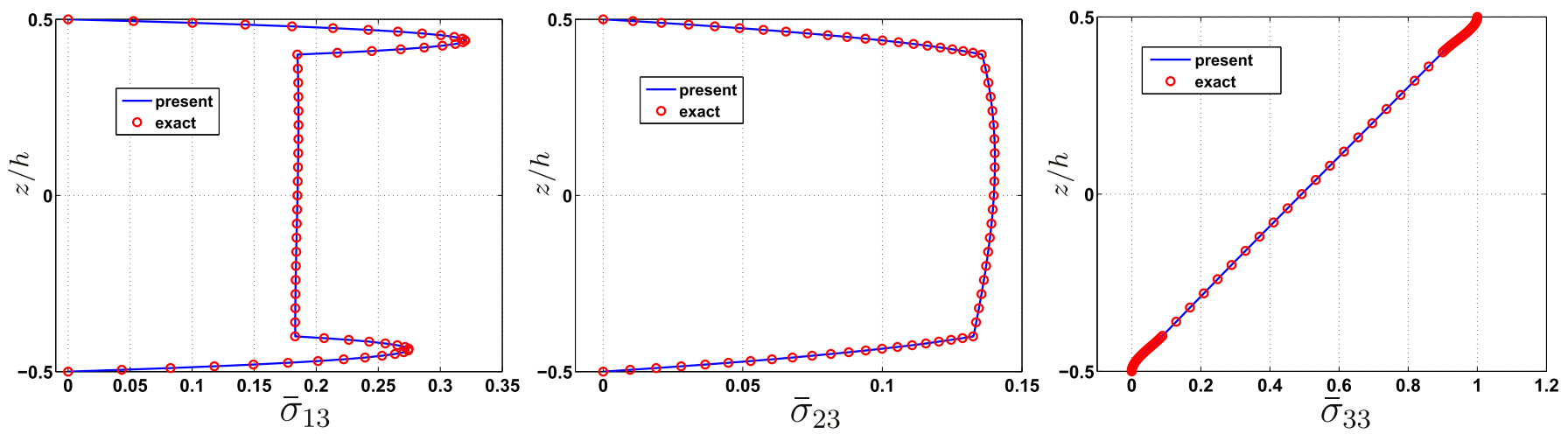

Fig. 7. Distribution of $\bar{\sigma}_{13}$ (left), $\bar{\sigma}_{23}$ (middle) and $\bar{\sigma}_{33}$ (right) along the thickness $-\mathrm{S}=2$ - sandwich.

- The transverse displacement $\bar{w}$ is not constant.

- The bending stress $\bar{\sigma}_{11}$ is not symmetric.

- As far as the transverse shear stress $\bar{\sigma}_{13}$ is concerned, the maximum value is localized in the face.

Finally, the top and bottom boundary conditions are fulfilled. In Table 4, the results are also compared with the LM4 approach [48] involving a Q9 plate element, and they are rather close.

\subsection{Bending analysis of symmetric plate under localized pressure}

In this section, the behavior of the approach is illustrated through a more severe test case. The ability of this one to capture local effects is shown. The results are also compared with the equivalent displacement-based approach involving variables separation [52]. In this latter, the fourth-order expansion with respect to the $\mathrm{z}$-coordinate is also considered.

This test is about simply-supported plate submitted to a localized pressure. It is shown in Fig. 8 and detailed below:

geometry: square composite cross-ply plate $\left(0^{\circ} / 90^{\circ} / 0^{\circ}\right), a=b$. All layers have the same thickness. $S \in\{4,40\}$

boundary conditions: simply-supported plate subjected to a localized pressure $q(x, y)=q_{0}$ applied on a square area with a size of $a / 10 \times b / 10$ at the plate center (see Fig. 8 right).

material properties: same material as in Section 4.1

mesh: $N_{x}=N_{y}=36$ with a space ratio of 5 (denoted $\operatorname{sr}(5)$ ) (see Fig. 8 left), a quarter of the plate is meshed.

number of dofs: $N d o f_{x y}=24198$ and $N d o f_{z}=24 \times N C+6=78$
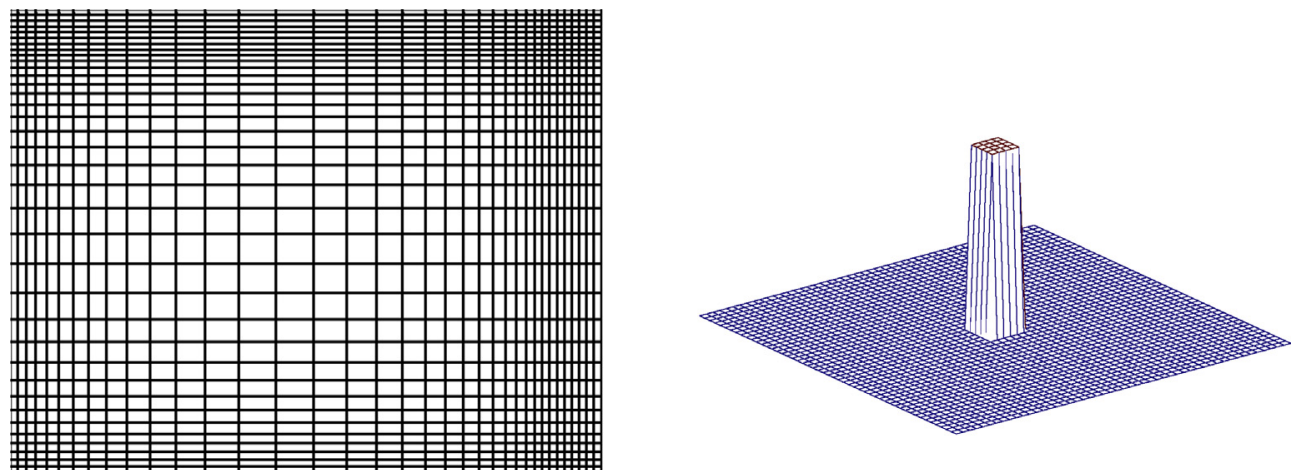

Fig. 8. Mesh of a quarter $N_{x}=N_{y}=36 \operatorname{sr}(5)$ (left) - localized pressure (right).
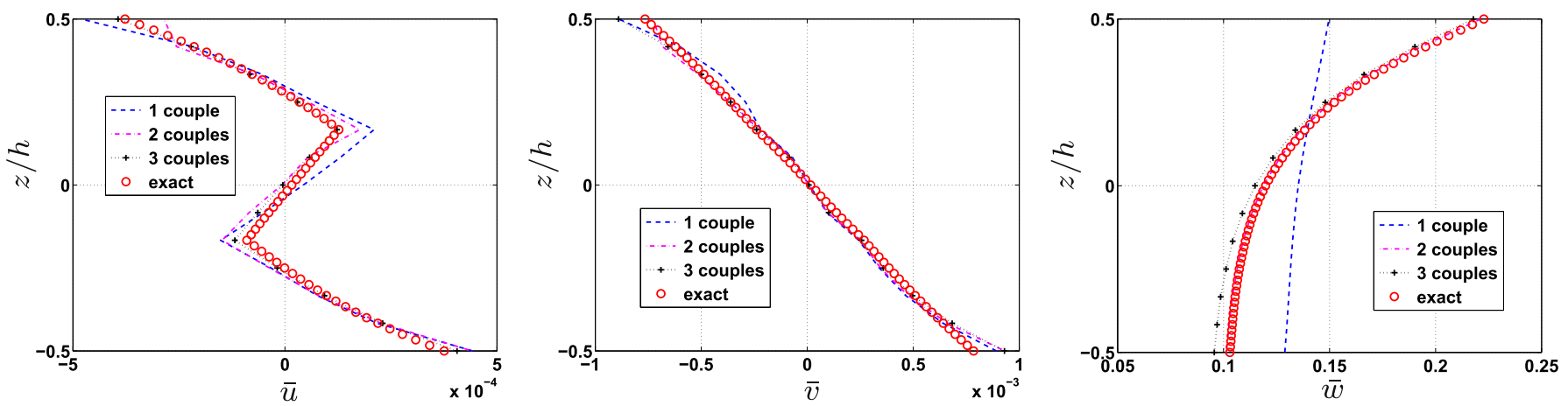

Fig. 9. Distribution of $\bar{u}$ (left), $\bar{v}$ (middle) and $\bar{w}$ (right) along the thickness $-\mathrm{S}=4-3$ layers - localized pressure. 

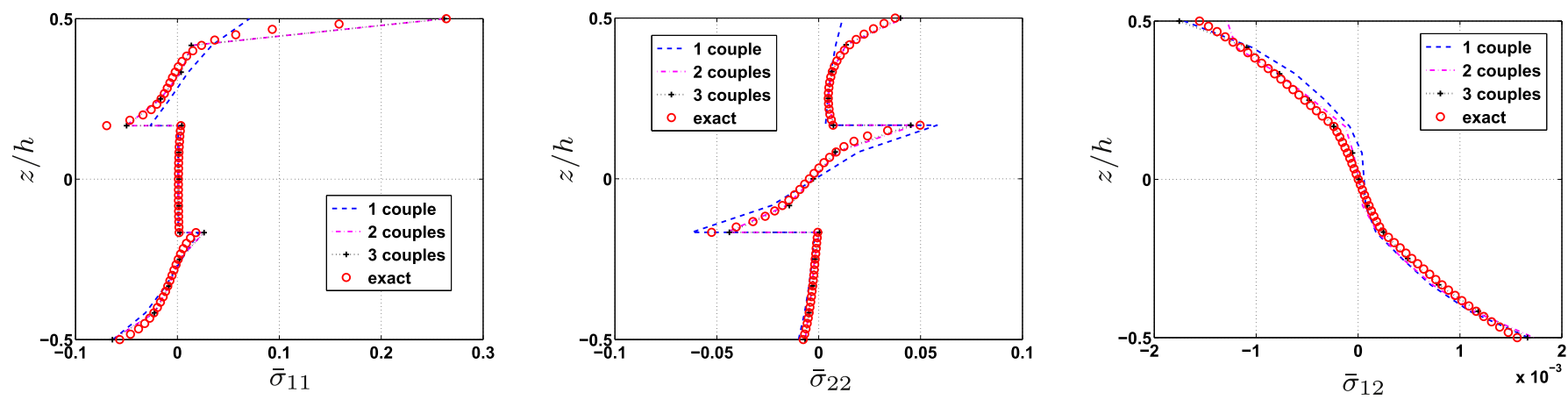

Fig. 10. Distribution of $\bar{\sigma}_{11}$ (left), $\bar{\sigma}_{22}$ (middle) and $\bar{\sigma}_{12}$ (right) along the thickness $-\mathrm{S}=4$ - 3 layers - localized pressure.
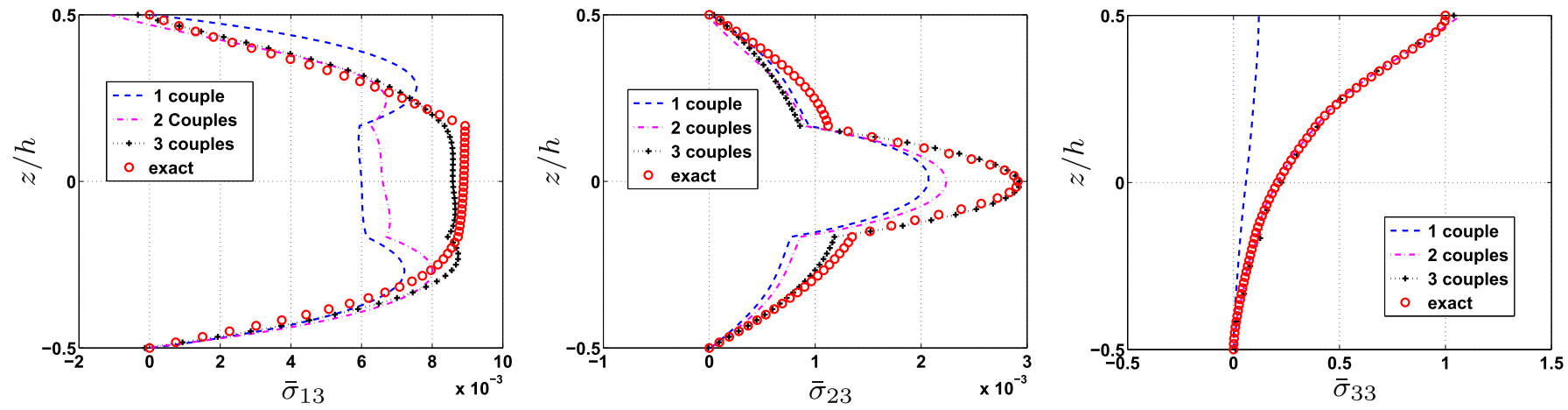

Fig. 11. Distribution of $\bar{\sigma}_{13}$ (left), $\bar{\sigma}_{23}$ (middle) and $\bar{\sigma}_{33}$ (right) along the thickness $-\mathrm{S}=4$ - 3 layers - localized pressure.

results: displacements and stresses are made non-dimensional as in Section 4.1.

reference values are obtained with 450 terms in the Fourier series ([53]). The applied pressure is shown in Fig. 8 right.

The distribution of displacements and stresses across the thickness are given in Figs. 9-11. We can notice that few couples allow us to recover a solution with a good accuracy:

- Only one or two couples are sufficient for the displacements (cf. Fig. 9) and for the in-plane and transverse normal stresses (cf. Figs. 10 and 11 right).

- A significant correction is brought by the third couple for the transverse shear stresses (cf. Fig. 11 left and middle).

Then, the construction of 15 additional couples implies minor corrections to derive the distributions of displacements and stresses in excellent agreement with the reference solution. See Figs. 12-14.

In Fig. 14, the distributions of the transverse stresses along the thickness of the present method are compared with the separated representation solution based on the principle of virtual displacements (denoted PVD 4th order [52]). The two approaches perform very well. The difference lies on the small discontinuity of the transverse stresses between two adjacent layers which is overcome by the mixed approach.

The distributions of the transverse stress along the thickness are also given for a thin plate $(S=40)$ in Fig. 15. The same number of couples is built. It can be inferred from this figure that the results agree very well with the reference solution. As in the previous examples, the boundary conditions on the upper and lower surfaces are fulfilled.
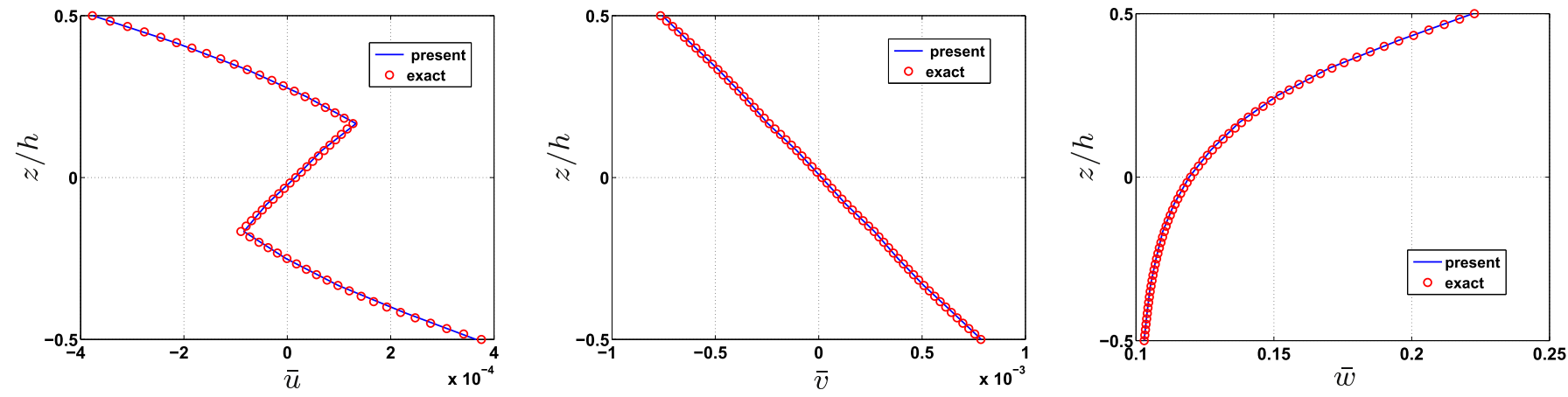

Fig. 12. Distribution of $\bar{u}$ (left), $\bar{v}$ (middle) and $\bar{w}$ (right) along the thickness $-\mathrm{S}=4-3$ layers - localized pressure. 

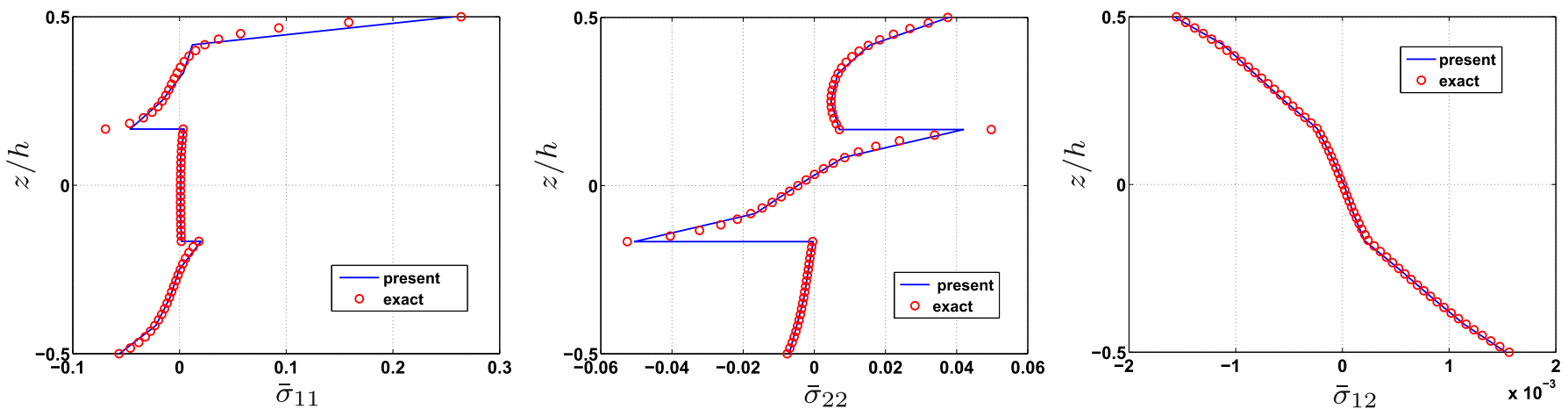

Fig. 13. Distribution of $\bar{\sigma}_{11}$ (left), $\bar{\sigma}_{22}$ (middle) and $\bar{\sigma}_{12}$ (right) along the thickness $-\mathrm{S}=4$ - 3 layers - localized pressure.
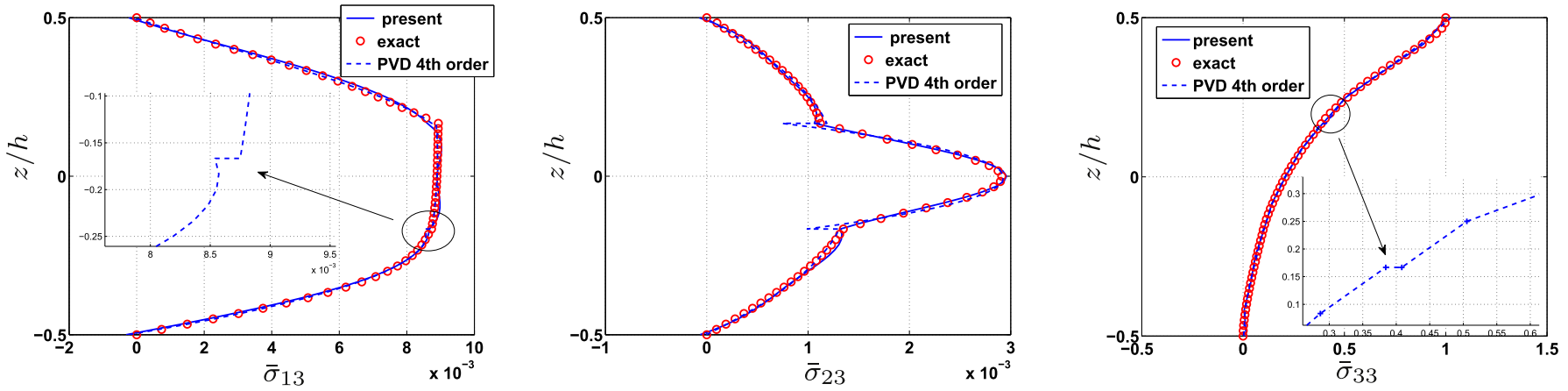

Fig. 14. Distribution of $\bar{\sigma}_{13}$ (left), $\bar{\sigma}_{23}$ (middle) and $\bar{\sigma}_{33}$ (right) along the thickness $-\mathrm{S}=4$ - 3 layers - localized pressure.
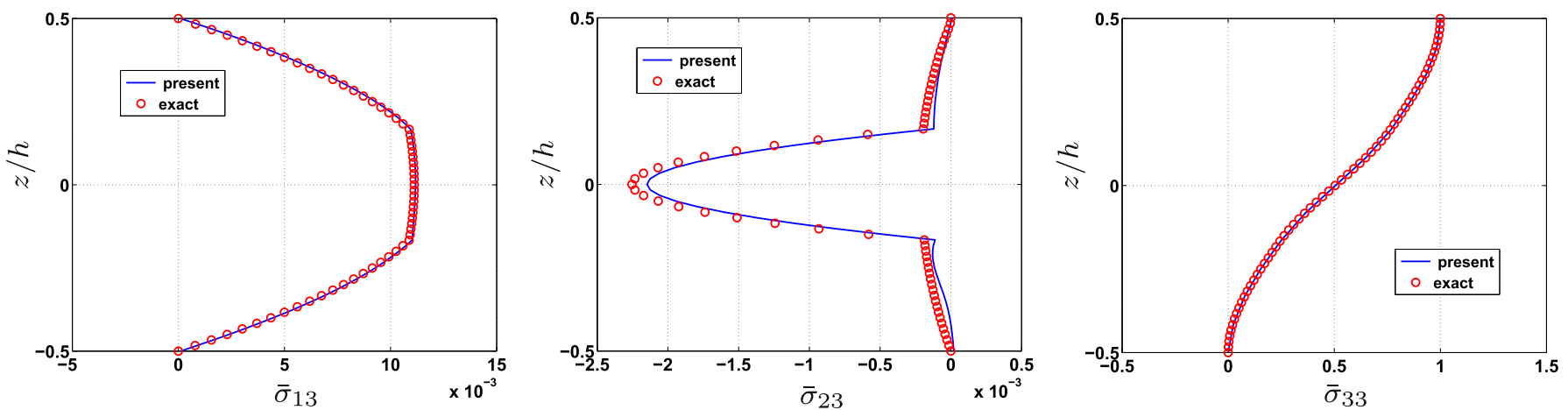

Fig. 15. Distribution of $\bar{\sigma}_{13}$ (left), $\bar{\sigma}_{23}$ (middle) and $\bar{\sigma}_{33}$ (right) along the thickness $-\mathrm{S}=40$ - 3 layers - localized pressure.

\subsection{The computational cost of this new approach}

In this section, the computational complexity of the present PGD method is addressed. A comparison with a layerwise approach is given.

By assuming the use of a direct band solver, the estimation of the number of operations, denoted $\mathrm{NbOp}$, gives:

- LayerWise Approach: $N b O p \sim 10^{5} N_{x}^{3} \times N_{y} \times D e g z^{3} \times N_{z}^{3}$

- PGD approach: $N b O p \sim 10^{3} N_{x}^{3} \times N_{y} \times N_{\text {couple }}$

where Degz is the order of expansion of the unknowns with respect to $z, N_{z}$ is the number of numerical layers, and $N_{\text {couple }}$ is the number of couples built in the PGD process. The cost of the 1D problem involving the $\mathrm{z}$ functions is neglected. We also assume that $N_{x}>N_{y}$. This estimation is suitable when the number of elements $N_{x} \times N_{y}$ and $N_{z}$ are high. It allows us to show the major influence of the number of layers and the z-expansion degree on the computational time of the LW approach. On the contrary, the PGD approach is not affected by these parameters. This is particularly interesting for the modeling of multi-layered composite structures that exhibit complex behavior in the thickness direction. So, the most important gain of the PGD approach will be made for complex problems where the number of physical layers increases, i.e. when $100 \mathrm{Degz}^{3} \times N_{z}^{3} \gg N_{\text {couple }}$. And, it seems that the number of couples can be low.

To illustrate the gain in terms of number of unknowns, a 24layered square plate $\left(0^{\circ} / 90^{\circ}\right)_{12}$ with the same test configuration 

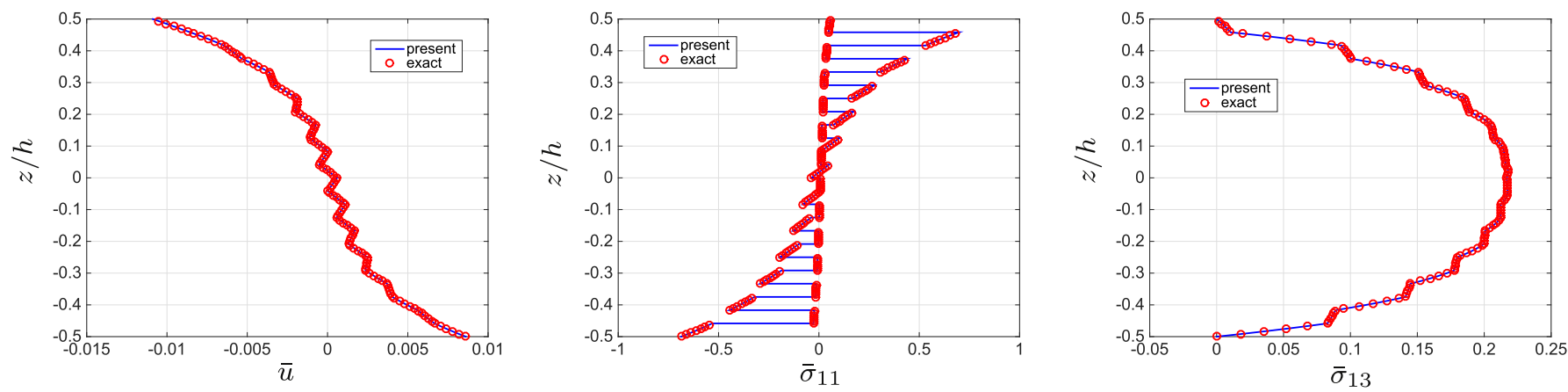

Fig. 16. Distribution of $\bar{u}_{1}$ (left), $\bar{\sigma}_{11}$ (middle) and $\bar{\sigma}_{13}$ (right) along the thickness $-\mathrm{S}=4-24$ layers.

as in Section 4.3 is considered. The total number of unknowns gives:

- LayerWise Approach: 1.062.150 dofs

- PGD approach: $N d o f_{x y}+N d o f_{z}=10.950+582$ dofs

The accuracy of the results are shown in Fig. 16 with one couple. The distribution of $\bar{u}, \bar{\sigma}_{11}$ and $\bar{\sigma}_{13}$ through the thickness is in very good agreement with the exact solution. The other results are not given for brevity reason. Short computational time (few seconds) on a standard laptop is needed to achieve this solution.

\section{Conclusion}

In this paper, variable separation in the framework of a partially Reissner's Mixed Variational Theorem is proposed for the modeling of laminated composite plates. A 8-node FE for the in-plane unknown approximation and a fourth-order LW description for the thickness unknown approximation are used. In this formulation, all interface conditions are exactly satisfied. The approach has been assessed on various stacking sequences, slenderness ratios, geometries and boundary conditions.

Comparisons with exact reference solutions, results available in open literature have shown the very good accuracy of the method with a reduced computational cost. In fact, the number of layers have no influence on the computational cost as only the cost of the 1D problem is affected by this number. This is particularly interesting in the framework of a mixed approach, where the number of unknowns involving both displacements and transverse stresses becomes very important in a classical LW method. So, the present work can provide quasi-3D results avoiding expensive 3D FEM or LW computations. Therefore, this method seems to have very attractive features.

\section{References}

[1] Tanigawa Y, Murakami H, Ootao Y. Transient thermal stress analysis of a laminated composite beam. J Therm Stress 1989;12:25-39.

[2] Yang P, Norris C, Stavsky Y. Elastic wave propagation in heterogeneous plates. Int J Solids Struct 1966;2:665-84.

[3] Lo K, Christensen R, Wu F. A higher-order theory of plate deformation. part ii: laminated plates. J Appl Mech ASME 1977;44:669-76.

[4] Reddy J. A simple higher-order theory for laminated composite plates. J Appl Mech ASME 1984;51(4):745-52.

[5] Carrera E. A priori vs. a posteriori evaluation of transverse stresses in multilayered orthotropic plates. Compos Struct 2000;48(4):245-60.

[6] Kant T, Swaminathan K. Analytical solutions for the static analysis of laminated composite and sandwich plates based on a higher order refined theory Compos Struct 2002;56:329-44.

[7] Aydogdu M. A new shear deformation theory for laminated composite plates. Compos Struct 2006;89(1):94-101.
[8] Pagano N. Exact solutions for composite laminates in cylindrical bending. J Comp Mater 1969;3:398-411.

[9] Reddy J. On refined computational models of composite laminates. Int J Num Meth Eng 1989;27:361-82.

[10] Robbins D, Reddy J. Modelling of thick composites using a layerwise laminate theory. Int J Num Meth Eng 1993;36:655-77.

[11] Ferreira A. Analysis of composite plates using a layerwise shear deformation theory and multiquadrics discretization. Mech Adv Mater. Struct 2005;12: 99-112.

[12] Icardi U. Higher-order zig-zag model for analysis of thick composite beams with inclusion of transverse normal stress and sublaminates approximations. Compos Part B Eng J 2001;32:343-54.

[13] Carrera E. A study of transverse normal stress effect on vibration of multilayered plates and shells. J Sound Vibr 1999;225:803-29.

[14] Reddy J. Mechanics of laminated composite plates and shells - theory and analysis. CRC Press Inc; 2004.

[15] Kapania R, Raciti S. Recent advances in analysis of laminated beams and plates. Part I: shear effects and buckling. AIAA J 1989;27:923-34.

[16] Noor A, Burton W. Assessment of computational models for multilayered composite shells. Appl Mech Rev 1990;43(4):67-97.

[17] Carrera E. Theories and finite elements for multilayered, anisotropic, composite plates and shells. Arch Comput Meth Eng 2002;9:87-140.

[18] Carrera E. Historical review of zig-zag theories for multilayered plates and shells. Appl Mech Rev 2003:56(3):287-308.

[19] Reddy J, Arciniega R. Shear deformation plate and shell theories: from stavsky to present. Mech Adv Mater. Struct 2004;11:535-82.

[20] Zhang Y, Yang C. Recent developments in finite elements analysis for laminated composite plates. Compos Struct 2009;88:147-57.

[21] Rolfes R, Rohwer K. Improved transverse shear stresses in composite finite elements based on first order shear deformations theory. Int J Num Meth Eng 1997; 40:51-60.

[22] Rolfes R, Rohwer K, Ballerstaedt M. Efficient linear transverse normal stress analysis of layered composite plates. Comput Struct 1998;68:643-52.

[23] Noor A, Burton W, Peters J. Predictor-corrector procedure for stress and free vibration analyses of multilayered composite plates and shells. Comput Methods Appl Mech Eng 1990;82:341-64.

[24] Kapuria S, Dumir P, Ahmed A. An efficient higher order zigzag theory for composite and sandwich beams subjected to thermal loading. Int J Solids Struct 2003;40:6613-31.

[25] Lee C-Y, Liu D, Lu X. Static and vibration analysis of laminated composite beams with an interlaminar shear stress continuity theory. Int J Num Meth Eng 1992;33:409-24.

[26] Sciuva MD, Icardi U. Numerical assessment of the core deformability effect on the behavior of sandwich beams. Compos Struct 2001;52:41-53.

[27] Vidal P, Polit O. A refined sinus plate finite element for laminated and sandwich structures under mechanical and thermomechanical loads. Comput Methods Appl Mech Eng 2013;253:396-412.

[28] Vidal P, Polit O, D'Ottavio M, Valot E. Assessment of the refined sinus plate finite element: free edge effect and Meyer-Piening sandwich test. Finite Elem Analysis Des 2014;92:60-71. http://dx.doi.org/10.1016/j.finel.2014.08.004.

[29] Mau S, Tong P, Pian T. Finite element solutions for laminated thick plates. J Comp Mater 1972;6:304-11.

[30] Jing H-S, Liao M. Partial hybrid stress element for the analysis of thick laminated composite plates. Int J Num Meth Eng 1989;28(12):2813-27.

[31] Vu-Quoc L, Tan X. Efficient hybrid-eas solid element for accurate stress prediction in thick laminated beams, plates, and shells. Comput Methods Appl Mech Eng 2013;253:337-55.

[32] Aitharaju V, Averill R. $C^{0}$ zig-zag kinematic displacement models for the analysis of laminated composites. Mech Compos Mater. Struct 1999;6: $31-56$.

[33] Gherlone M, Sciuva MD. Thermo-mechanics of undamaged and damaged multilayered composite plates: a sub-laminates finite approach. Comput Struct 2007;81:125-36. 
[34] Vidal P, Polit O. A refined sine finite element with transverse normal stress for thermoelastic analysis of laminated composite in cylindrical bending. J Therm Stress 2011;34(11):1185-204.

[35] Cook G, Tessler A. A $\{3,2\}$-order bending theory for laminated composite and sandwich beams. Compos Part B Eng J 1998;29B:565-76.

[36] Ramtekkar G, Desai Y, Shah A. Mixed finite-element model for thick composite laminated plates. Mech Adv Mater. Struct 2002;9:133-56.

[37] Moleiro F, Soares CM, Soares CM, Reddy J. Mixed least-squares finite element models for static and free vibration analysis of laminated composite plates. Comput Methods Appl Mech Eng 2009;198:1848-56.

[38] Moleiro F, Soares CM, Soares CM, Reddy J. Layerwise mixed least-squares finite element models for static and free vibration analysis of multilayered composite plates. Compos Struct 2010;92:2328-38.

[39] Pagano NJ. Stress fields in composite laminates. Int J Solids Struct 1978;14: $385-400$.

[40] Thai N, D'Ottavio M, Caron J-F. Bending analysis of laminated and sandwich plates using a layer-wise stress model. Compos Struct 2013;96:135-42.

[41] Reissner E. On a certain mixed variational theorem and a proposed application. Int J Num Meth Eng 1984;20:1366-74.

[42] Reissner E. On a mixed variational theorem and on a shear deformable plate theory. Int J Num Meth Eng 1986;23:193-8.

[43] Murakami H. Laminated composite plate theory with improved in-plane responses. J Appl Mech ASME 1986;53:661-6.

[44] Toledano A, Murakami H. A high-order laminated plate theory with improved in-plane responses. Int J Solids Struct 1987;23:111-31.

[45] Toledano A, Murakami H. A composite plate theory for arbitrary laminate configurations. J Appl Mech ASME 1987;24:181-9.

[46] Carrera E. Evaluation of layerwise mixed theories for laminated plates analysis. AIAA J 1998;36(5):830-9.
[47] Carrera E, Demasi L. Classical and advanced multilayered plate elements based upon pvd and rmvt. part 1: derivation of finite element matrices. Int J Num Meth Eng 2002;55:191-231.

[48] Carrera E, Demasi L. Classical and advanced multilayered plate elements based upon pvd and rmvt. part 2: numerical implementations. Int J Num Meth Eng 2002;55:253-91.

[49] Carrera E. Developments, ideas and evaluations based upon the reissner's mixed theorem in the modeling of multilayered plates and shells. Appl Mech Rev 2001;54:301-29.

[50] Ammar A, Mokdada B, Chinesta F, Keunings R. A new family of solvers for some classes of multidimensional partial differential equations encountered in kinetic theory modeling of complex fluids. J Newt Fluid Mech 2006;139:153-76.

[51] Bognet B, Bordeu F, Chinesta F, Leygue A, Poitou A. Advanced simulation of models defined in plate geometries: $3 \mathrm{~d}$ solutions with $2 \mathrm{~d}$ computational complexity. Comput Methods Appl Mech Eng 2012;201-204:1-12. http:/ dx.doi.org/10.1016/j.cma.2011.08.025.

[52] Vidal P, Gallimard L, Polit O. Proper generalized decomposition and layer-wise approach for the modeling of composite plate structures. Int J Solids Struct 2013;50(14-15):2239-50. http://dx.doi.org/10.1016/j.ijsolstr.2013.03.034.

[53] Pagano N. Exact solutions for rectangular bidirectional composites and sandwich plates. J Comp Mater 1970;4:20-34.

[54] D'Ottavio M, Ballhause D, Wallmersperger T, Kröplin B. Considerations on higher-order finite elements for multilayered plates based on a unified formulation. Comput Struct 2006;84:1222-35.

[55] Liou W-J, Sun C. A three-dimensional hybrid stress isoparametric element for the analysis of laminated composite plates. Comput Struct 1987;25:241-9.

[56] Wu Z, Chen R, Chen W. Refined laminated composite plate element based on global-local higher-order shear deformation theory. Compos Struct 2005;70: 135-52. 